\title{
Bio-directed identification of untargeted toxicants in industrial wastewater guides upgrading water treatments
}

Jing Guo ${ }^{a}$ Dongyang Denga, Jingfan Qiub, Jinyou Shene, Lianjun Wange, Si Weia, Xiaowei Zhang ${ }^{\mathrm{a}}$, Qing Zhou ${ }^{\mathrm{a},{ }^{*}}$, Hongxia Yu${ }^{\mathrm{a}}$, Wei Shi ${ }^{\mathrm{a}, \mathrm{c}, \mathrm{d}}$

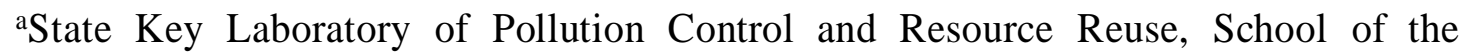
Environment, Nanjing University, Nanjing, Jiangsu 210023, China

bKey Laboratory of Pathogen Biology of Jiangsu Province, Department of Pathogen Biology, Nanjing Medical University, Nanjing 211166, China

cJiangsu Key Laboratory of Environmental Safety and Health Risk of Chemicals, Nanjing, Jiangsu 210023, China

${ }^{\mathrm{d}}$ Research Center for Environmental Toxicological \& Safety of Chemicals, Nanjing University, Nanjing 210023, China

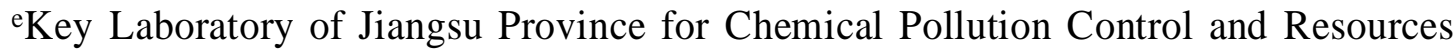
Reuse, School of Environmental and Biological Engineering, Nanjing University of Science and Technology, Nanjing 210094, China

\section{CORRESPONDING AUTHOR}

Qing Zhou (zhouqing@nju.edu.cn)

\section{Supporting Information}

This file includes:

Pages: S1-S33, Figures: S1-S6, Tables: S1-S7 


\section{Preparation of wastewater samples}

Four liters of samples were collected after filtering using a glass fiber filter $(0.7 \mu \mathrm{m}$, Whatman) and a nylon filter $(0.45 \mu \mathrm{m}$, Whatman). Further extraction of the wastewater samples was performed on solid-phase extraction (SPE) cartridges (6 cc/500 mg, Waters Oasis HLB), which was preconditioned with hexane (Hex), dichloromethane (DCM) and methanol $(\mathrm{MeOH})$, via vacuum extraction at 1-2 drops/s. Each cartridge was used for extraction of the $500 \mathrm{~mL}$ wastewater samples, which were further centrifuged at $4616 \times \mathrm{g}$ for $5 \mathrm{~min}$ to remove the water content. The dried cartridges were eluted with $5 \mathrm{~mL}$ of Hex, $10 \mathrm{~mL}$ of $\mathrm{DCM}$ and $10 \mathrm{~mL}$ of $\mathrm{MeOH}$ in series. The elution was concentrated to $1 \mathrm{~mL}$ by rotary vacuum evaporation followed by concentration under gentle nitrogen, and stored at $-20^{\circ} \mathrm{C}$ until analysis.

\section{Toxicity evaluation by algae, zebrafish embryos and Daphnia Magna}

Toxicity to green algae. The acute toxicity tests were conducted according to the Environmental Protection Agency standard procedures ${ }^{1}$. All tests were carried out in an air-conditioned light incubator with temperature of $28 \pm 0.5^{\circ} \mathrm{C}$ and photoperiod of 12 h light:12 h dark. Algal cells in the exponential phase of growth were inoculated in 50 mL Erlenmeyer flasks containing $20 \mathrm{~mL}$ of WC medium at the initial concentration of $7 \times 10^{4}$ cells/mL. Then the biomass was measured at 24, 48, 72 and $96 \mathrm{~h}$ after exposure. Since the most suitable wavelength for monitoring algal growth was $485 \mathrm{~nm}$ and a good linear relationship exists between cell number and chlorophyll a content of alga, the algal biomass can be calculated indirectly using spectrophotometric data. 
WC medium containing S. obliquus cells were distributed to $50 \mathrm{~mL}$ Erlenmeyer flasks with different concentrations of wastewater, and incubated for $96 \mathrm{~h}$ in a light incubator. Five gradients of concentrations were prepared in each test followed dilution ratios of 2, 4, 8, 16 and 32. Four replicates were made for all treatment groups and controls (WC medium), and independent experiments were performed at triplicate. All algal cultures grew under the same conditions as described above. For S. obliquus, the absorbance of cultures was measured after the algae were treated for 24, 48, 72, and 96 h. Inhibition percentages related to the growth of control systems were then calculated. The acute toxicity of wastewater on algae was assessed by calculating the median effect concentration values of the treated groups, and the EC50 was expressed as the median concentration causing 50\% reduction of the algal cell density.

Toxicity to zebrafish embryos. Danio rerio was originally obtained from the Institute of Hydrobiology, Chinese Academy of Sciences and were selected in the pure culture in the laboratory. Light was controlled with a timer to provide a 14-h light/10-h dark cycle; temperature was maintained at $27 \pm 1^{\circ} \mathrm{C}$. Conductivity was at $500 \mu \mathrm{S}$. Males and females were continuously held together at a 1:1 ratio in $5 \mathrm{~L}$ tanks with a maximum of 4 fish per liter. Animals were optimally fed with thawed Artemia larvae (LandmanHoevelaken). Three days before the start of the experiments, the 12-month-old adults were separated. One day before spawning, adults were placed in a tank with a grid bottom. Light regime was adjusted to obtain eggs timely in the proper embryonic phase (4-32 cell stage-blastomeres) for testing. Thirty minutes after the onset of spawning 
the eggs were collected with a glass siphon. Unfertilized (opaque) eggs were separated from fertilized eggs.

The test method followed the draft OECD protocol "fish embryo toxicity" (FET) test ${ }^{2}$. Following a screening test for the selection of test concentrations and the development of abnormalities in the embryos, the exposure time of the final test was chosen to be $96 \mathrm{~h}$. To simultaneously start the exposure, 40 fertilized eggs were brought in $50 \mathrm{~mL}$ test solution. Viable eggs were selected and incubated in 24-well plates. Eggs were individually incubated in $2 \mathrm{ml}$ medium. Per plate, four wells were used for the control and 20 wells for a test concentration (instead of two independent replicates of 10 eggs per concentration). Plates were covered with Parafilm ${ }^{\circledR}$ and a lid to prevent evaporation during exposure. Temperature during exposure was kept at $26 \pm 1{ }^{\circ} \mathrm{C}$. The $\mathrm{pH}$ of the dilution medium (DSW) was on average 8.07 (range 7.7-8.1). Oxygen during exposure was $\geq 6.6 \mathrm{mg} / \mathrm{l}$. Embryos were exposed under static conditions and microscopic observations were done at 24, 48, 72 and $96 \mathrm{~h}$ after the start of the experiments under a Wild-binocular microscope 10-60×. Endpoints were: coagulation of embryo, tail detachment, absence of heartbeat, abnormal pigmentation, unsuccessful hatching, head-, heart-, tail-, yolk sac- and otholith deformations, and scoliosis and growth retardation.

Toxicity to Daphnia Magna. Newly hatched Daphnia magna (6-24 h) was used. Pretests were conducted to obtain a suitable concentration range, which ranged from the concentration with no inhibition to the concentration with $100 \%$ inhibition. Five individuals and $10 \mathrm{~mL}$ of the test solutions or blanks were exposed in each glass beaker. 
The test solutions were solvent change to dimethyl sulfoxide (DMSO) in advance, using a DMSO content of no more than 5\%. A concentration gradient prepared by dilution ratios of 2, 4, 8, 16 and 32 was applied in each test. Four duplicates were conducted for each concentration. The temperature was maintained at $25 \pm 1{ }^{\circ} \mathrm{C}$ during the tests, with a light cycle of $14 \mathrm{~h}$ of light and $10 \mathrm{~h}$ of darkness. A positive control (reference substance, potassium dichromate, $\mathrm{K}_{2} \mathrm{CrO}_{4}$ ) and negative control (dilution water) were conducted for each test. Immobilization, shown as no response to gentle agitation, of the test species was used to determine the $50 \%$ effective concentration (EC50). The EC50 of the reference toxicant, $\mathrm{K}_{2} \mathrm{CrO}_{4}$, to Daphnia magna was $1.3 \pm 5.0 \times 10^{-2} \mathrm{mg} / \mathrm{L}$, which met the requirement for a valid test suggested by ISO $6431^{3}$.

\section{Toxicity identification and evaluation of the industrial wastewater}

pH adjustment: to characterize toxicity contribution of substances susceptible to acidity and alkalinity. Acidity and alkalinity of wastewater was adjusted by $\mathrm{pH}$ adjustment solutions $(1.0 \mathrm{~mol} / \mathrm{L} \mathrm{NaOH}, 0.1 \mathrm{~mol} / \mathrm{L} \mathrm{NaOH}, 1.0 \mathrm{~mol} / \mathrm{L} \mathrm{HCl}, 0.1 \mathrm{~mol} / \mathrm{L}$ $\mathrm{HCl}$ ). The industrial wastewater was divided into 3 parts, each with $1 \mathrm{~L}$. The $\mathrm{pH}$ of two of them was adjusted to 3 and 11, respectively, with the $\mathrm{pH}$ of the rest remained the same as original. Samples with $\mathrm{pH} 3$ and $\mathrm{pH} 11$ were adjusted back to $\mathrm{pH}$ i after being placed for a while. The $\mathrm{pH}$ of the wastewater samples was re-evaluated after kept at $4{ }^{\circ} \mathrm{C}$ overnight, which was re-adjusted to $\mathrm{pH}$ i when $\mathrm{pH}$ variations were observed.

Aeration: to characterize toxicity contribution of volatile or easily oxidized substances. $200 \mathrm{~mL}$ of $\mathrm{pH}$ adjusted wastewater (with individual $\mathrm{pH}$ of 3, i and 11) was 
aerated for an hour. The aeration device should be submerged under the water. The $\mathrm{pH}$ variations were also monitored through the aeration process. The $\mathrm{pH}$ of the wastewater was monitored every 5 minutes in the first 30 minutes, while was monitored every 10 minutes in the last 30 minutes. If the $\mathrm{pH}$ varied over 0.2 units, the $\mathrm{pH}$ of the water will be re-adjusted to $\mathrm{pH}$ i. The $\mathrm{pH}$ of the wastewater samples was re-evaluated after kept at $4{ }^{\circ} \mathrm{C}$ overnight, which was re-adjusted to $\mathrm{pH}$ i when $\mathrm{pH}$ variations were observed.

HLB solid-phase extraction (SPE): to characterize toxicity contribution of organic substances extracted by SPE on HLB columns. HLB columns were firstly activated by hexane, dichloromethane, methanol and pure water, respectively. Then, $200 \mathrm{~mL}$ of $\mathrm{pH}$ adjusted wastewater (with individual $\mathrm{pH}$ of 3, i and 11) was extracted by activated HLB SPE columns (6 cc/500 mg, Waters Oasis HLB). $100 \mathrm{~mL}$ of the wastewater which went through HLB columns were collected. The $\mathrm{pH}$ of these collected wastewaters, which were extracted by SPE in advance, was adjusted to their original $\mathrm{pH}$ values (ph 3, i, 11, respectively). The $\mathrm{pH}$ of the wastewater samples was reevaluated after kept at $4{ }^{\circ} \mathrm{C}$ overnight, which was re-adjusted to their original pHs when $\mathrm{pH}$ variations were observed.

EDTA chelation: to characterize toxicity contribution of metal cations. EDTA was added into the wastewater in gradient. More specific, 0, 0.0125, 0.025, 0.05, 0.1, 0.2, $0.4 \mathrm{~mL}$ of EDTA stock solutions were added into the wastewater respectively. The concentration of EDTA should not exceed the sensitivity of the test organisms.

Gradient pH adjustment: to characterize toxicity contribution of substances easily affected by $\mathrm{pH}$ conditions, such as ammonia, sulfides, cyanides, ionized 
pesticides and some metals. The $\mathrm{pH}$ of the wastewater was adjusted by $\mathrm{pH}$ adjustment reagents to $\mathrm{pH} 6,7$ and 8 , respectively.

Reduction by $\mathrm{Na}_{2} \mathrm{~S}_{2} \mathrm{O}_{3}$ : to characterize toxicity contribution of oxidizing substances such as chlorine, bromine, iodine, divalent manganese ions and so on. More concretely, $0,0.2,0.4,0.6,0.8,1.0 \mathrm{~mL}$ of $\mathrm{Na}_{2} \mathrm{~S}_{2} \mathrm{O}_{3}$ stock solution was added into wastewater samples, respectively. The concentration of the $\mathrm{Na}_{2} \mathrm{~S}_{2} \mathrm{O}_{3}$ stock solution was $220 \mathrm{mg} / \mathrm{L} . \mathrm{Na}_{2} \mathrm{~S}_{2} \mathrm{O}_{3}$ was toxic to Daphnia magna, with a $50 \%$ lethal concentration of $22 \mathrm{mg} / \mathrm{L}$. Usually, the concentration of the $\mathrm{Na}_{2} \mathrm{~S}_{2} \mathrm{O}_{3}$ stock solution was 10 times the $50 \%$ lethal concentration of $\mathrm{Na}_{2} \mathrm{~S}_{2} \mathrm{O}_{3}$.

\section{Sample fractionation}

Preparative fractionation of the sample was performed by reversed-phase (RP) separation with a flow rate of $5 \mathrm{~mL} / \mathrm{min}$ on a Waters XBridge C18 column $(19 \mathrm{~mm} \times$ $150 \mathrm{~mm}, 5 \mu \mathrm{m}$ ) by semipreparative high-performance liquid chromatography (HPLC) (Waters AutoPurification), and a water-methanol gradient of 50:50 at 0 min to 0:100 at 50 min and held until 80 min was applied. The sample was fractionated into 11 fractions based on the peak distribution of UV absorption spectrum, and the collection time is shown in Table S4. Preparative fractions were extracted 3 times by dichloromethane (DCM). The extractions were collected, gently nitrogen-blew to $1 \mathrm{~mL}$ and solventexchanged to methanol for further chemical analysis and toxicity evaluation.

\section{Identification and quantitation of causative toxicants}


Chemical analyses. Chemical analyses were performed on an HPLC system (Agilent 1260, Agilent, USA) coupled to a quadrupole time-of-flight mass spectrometry (QTOF-MS) system (TripleTOF 5600, AB Sciex, USA). Liquid separation was performed on an Agilent C18 column $(2.1 \times 150 \mathrm{~mm}, 2.5 \mu \mathrm{m})$ at a flow rate of 300 $\mu \mathrm{L} / \mathrm{min}$ and a column temperature of $30{ }^{\circ} \mathrm{C}$. The gradient (water with 5 vol\% acetonitrile/acetonitrile) was 100:0 at $0 \mathrm{~min}$ and maintained for $1 \mathrm{~min}$, followed by 50:50 at $15 \mathrm{~min}$, then changed to 0:100 at $25 \mathrm{~min}$ and held until $30 \mathrm{~min}$, then 100:0 at $30.5 \mathrm{~min}$ and held until $40 \mathrm{~min}$. Chemical profiles were acquired in full scan mode from m/z 100 to $m / z 1250$ by information-dependent acquisition with electrospray ionization (ESI) in positive and negative modes. A QTOF external calibration was performed every 5 samples with an atmosphere pressure chemical ionization (APCI) Positive/Negative Calibration Solution to maintain the mass accuracy threshold less than 5 ppm. Qualitative analyses were processed with PeakView ${ }^{\mathrm{TM}}$ (Foster City, CA).

Identification of causative toxicants. Key toxicants in toxic fractions were identified by untargeted screening which was applied by formula generation, structure elucidation and TP prediction in series. More concretely, formulae were generated based on mass characteristics by limiting mass error, element amounts and isotopic difference. Structures acquired by in silico fragmentation were further elucidated based on chromatography characteristics by use of the in-house quantitative structureretention relationship (QSRR), toxicity characteristics as the predicted 48-h acute toxicity to Daphnia magna and commercial importance evaluated by references and patent links in Pubchem. TPs were searched in the publications and predicted by the 
Swiss federal institute of aquatic science and technology (EAWAG) biocatalysis/biodegradation database and pathway prediction system (EAWAG$\mathrm{BBD} / \mathrm{PPS})$.

For formula generation.

Identification of characteristic toxicants was performed using PeakView ${ }^{\mathrm{TM}}$. Peaks were prioritized by limiting peak area threshold (area $>1 \times 10^{4}$ ) and PLS analysis (variable importance of projection, VIP>1.0) after eliminating the fractionation blanks. Once a peak was identified in one fraction, it will not be prioritized in the following fractions. Based on mass characteristics, formulae were calculated by Formula Finder (PeakView ${ }^{\mathrm{TM}}$, Foster City, CA) after limiting mass error threshold to $5 \mathrm{ppm}$, signal-tonoise ratio $(\mathrm{S} / \mathrm{N})$ greater than 10 , element amounts as C50H200N10O10P5S5Cl10Br10F10 and isotopic difference lower than 20\%. Based on the acquired formulae and spectra, tentative structures were acquired by in silico fragmentation using MetFrag with a fragmentation score greater than 0.7.

For structure elucidation.

Chromatography characteristics were first evaluated where tentative structures were elucidated based on a developed in-house QSRR model. More concretely, the $\log _{\text {Kow }}$ range of toxic fractions was calculated by the developed linear relationship of $\log _{\text {Kow }}=0.1151$ RT -0.2162 , where $\log _{\text {Kow }}$ reflects hydrophobicity of tentative structure and RT is the retention time of tentative structure on chromatography. A relative error of $20 \%$ was applied to calculate the $\log _{\text {Kow }}$ range of individual fractions. False positive structures with logkow out of the hydrophobicity range of individual fractions were 
elucidated. Tentative structures were further elucidated by toxicity characteristics based on the 48-h acute toxicity to Daphnia magna of tentative structures, which were predicted by ECOSAR (EPI Suite, 4.0). Structures with the toxicity ranked in the top 3 for each formula were selected for further evaluation of commercial importance. The commercial importance of tentative structures was evaluated by searching patent links in Pubchem. Only structures with available patent links were retained as identified toxicants. Identified toxicants with patents records greater than 100 were considered as commercially important and were selected for further quantitative confirmation.

For TP prediction.

Firstly, TPs of the identified toxicants were searched in the publications. In addition, TPs were also predicted by EAWAG-BBD/PPS, which is a TP prediction system based on microbial biocatalytic reactions and biodegradation pathways. Among TPs predicted by EAWAG-BBD/PPS, only results of the initial three transformation reactions were retained. Obtained TPs were identified in toxic fractions by limiting mass accuracy lower than 5 ppm and isotopic difference lower than 20\%.

Quantitation of causative toxicants. Quantitation of the 6 identified toxicants was performed on an HPLC system (Agilent 1260, Agilent, USA) coupled with a triple quadrupole mass spectrometer (API 4000, AB Sciex, USA) with a Waters ACQUITY BEH C18 column $(2.1 \mathrm{~mm} \times 50 \mathrm{~mm}, 2.5 \mu \mathrm{m})$. Liquid separation was performed at a flow rate of $400 \mu \mathrm{L} / \mathrm{min}$ and a column temperature of $40^{\circ} \mathrm{C}$. The gradient (water /methanol) was $90: 10$ at $0 \mathrm{~min}$, kept at $0.5 \mathrm{~min}$, to $30: 70$ at $2 \mathrm{~min}$, to $20: 80$ at $4 \mathrm{~min}$, to $10: 90$ at 8 
min, to 90:10 at $8.5 \mathrm{~min}$ and held until $12 \mathrm{~min}$. Quantitative ions and the limit of detection (LOD) of causative toxicants were shown in Table S4. 
Figure S1 The scheme of wastewater treatments of FD WWTP in JT-CIP.

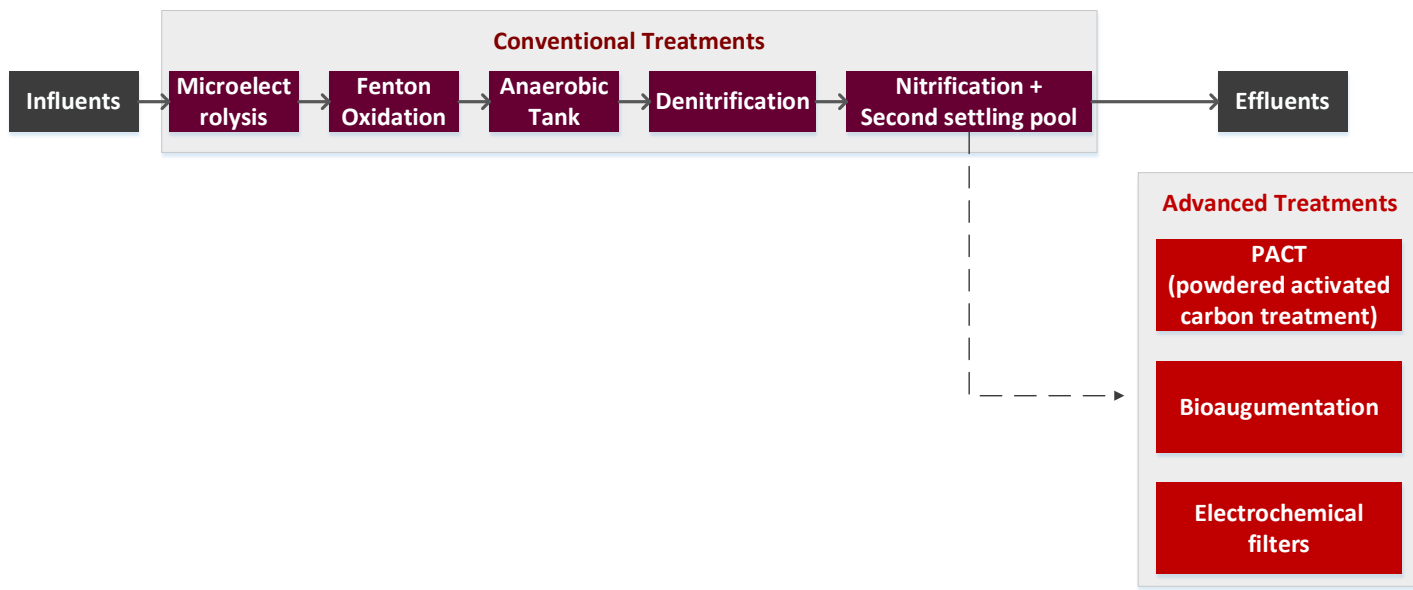


Figure S2 Recovery of artificial standards by HLB extraction

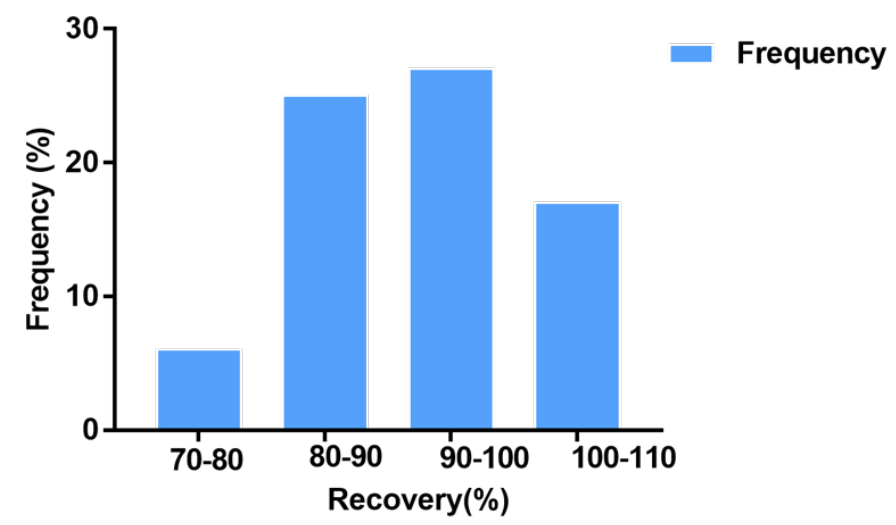


Figure S3 The scheme of toxicity identification and evaluation (TIE).

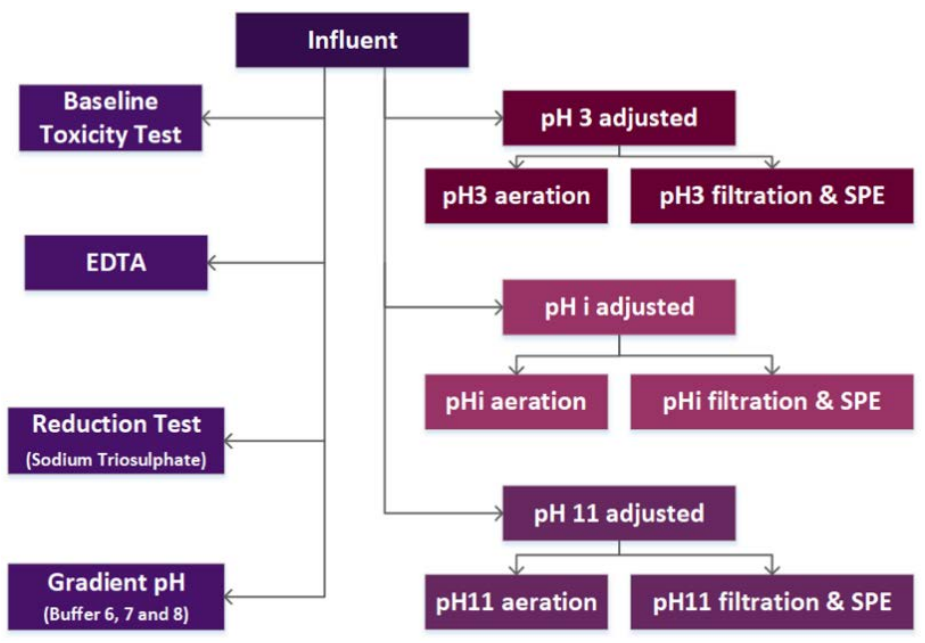


Figure S4. Identification of toxic components and toxic fractions.

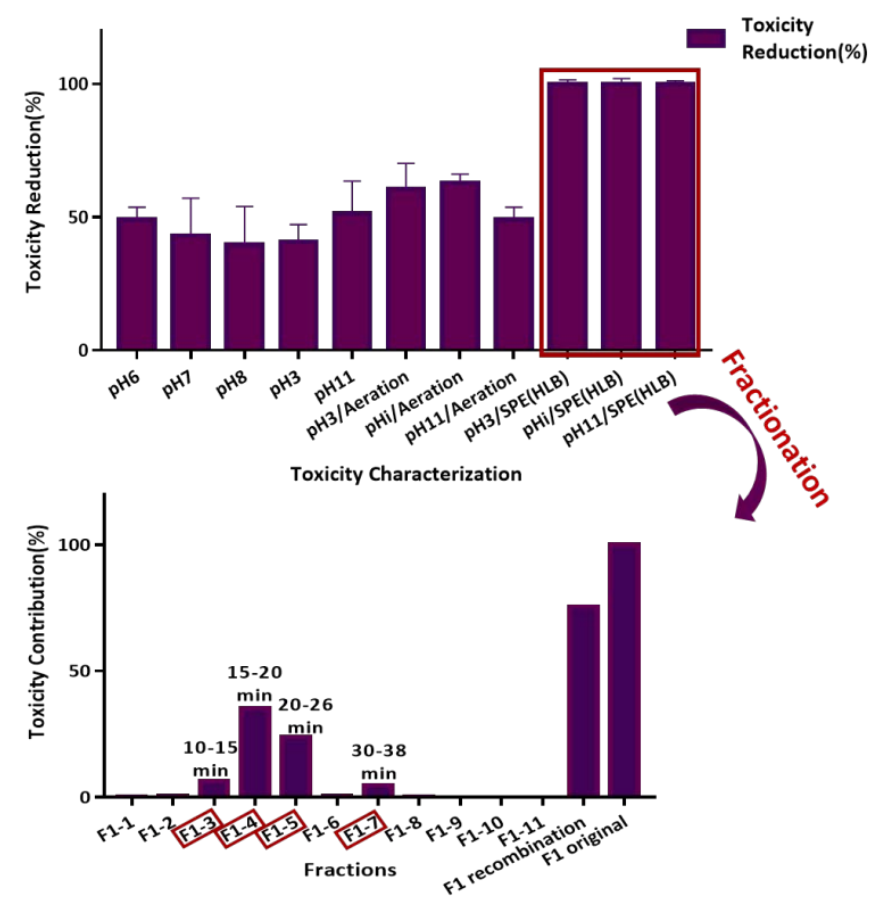


Figure S5. Qualitative identification of causative toxicants in toxic fractions.

\begin{tabular}{|c|c|c|c|c|c|c|}
\hline \multicolumn{3}{|c|}{ 1.Peaks Prioritization } & & & & \\
\hline \multirow{9}{*}{$\begin{array}{l}\text { Area }>1 \times 10^{4} \\
\& \text { \& } \&(V I P>1)\end{array}$} & FI'-3. & F14 & F1-5 & F1-7 & & \\
\hline & \multirow{2}{*}{$\begin{array}{l}190.0426 \\
165.0479\end{array}$} & 281.1227 & 257.1349 & 308.1521 & & \\
\hline & & 302.1097 & 274.2737 & \multirow{2}{*}{$\begin{array}{l}342.0770 \\
314.0817\end{array}$} & & \\
\hline & \multirow{2}{*}{ 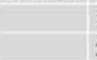 } & 198.1595 & \multirow{2}{*}{375.1333} & & & \\
\hline & & 204.0587 & & 314.0817 & & \\
\hline & \multicolumn{2}{|c|}{217.0535} & & & & \\
\hline & \multirow{2}{*}{\multicolumn{2}{|c|}{$\begin{array}{l}224.0037 \\
226.0405\end{array}$}} & & & & \\
\hline & & & & & & \\
\hline & 282.1440 & 242.9577 & & & & \\
\hline \multirow{2}{*}{\multicolumn{7}{|c|}{ 2.Formula Generation }} \\
\hline & & & & & & \\
\hline \multirow{15}{*}{$\begin{array}{l}\text { Formula } \\
\text { a) Accurate } \\
\text { Mass; } \\
\text { b) Element } \\
\text { Amounts; } \\
\text { e) Isotopic } \\
\text { Difrerence; } \\
\text { d) S/N Ratio; }\end{array}$} & \multicolumn{2}{|c|}{ F13 } & \multicolumn{2}{|c|}{ F1-4 } & F1-5 & F1-7. \\
\hline & \multicolumn{2}{|c|}{$\mathrm{C}_{9} \mathrm{H}_{2} \mathrm{~N}_{3} \mathrm{~S}$} & \multicolumn{2}{|c|}{$\mathrm{C}_{15} \mathrm{H}_{21} \mathrm{ClN}_{2} \mathrm{O}$} & $\mathrm{C}_{9} \mathrm{H}_{16} \mathrm{~N}_{6} \mathrm{O}_{3}$ & $\mathrm{C}_{16} \mathrm{H}_{2} \mathrm{ClN}_{3} \mathrm{O}$ \\
\hline & \multicolumn{2}{|c|}{$\mathrm{C}_{11} \mathrm{H}_{3} \mathrm{CIN}$} & \multicolumn{2}{|c|}{$\mathrm{C}_{12} \mathrm{H}_{22} \mathrm{ClFN}_{2} \mathrm{O}_{2}$} & $\mathrm{C}_{16} \mathrm{H}_{3 \mathrm{~N}} \mathrm{NO}_{2}$ & $\mathrm{C}_{13} \mathrm{H}_{23} \mathrm{ClFN}_{3} \mathrm{O}_{2}$ \\
\hline & \multicolumn{2}{|c|}{$\mathrm{C}_{8} \mathrm{H}_{9} \mathrm{CIFNO}$} & \multicolumn{2}{|c|}{$\mathrm{C}_{16} \mathrm{H}_{13} \mathrm{~F}_{2} \mathrm{~N}_{3} \mathrm{O}$} & $\mathrm{C}_{17} \mathrm{H}_{9} \mathrm{ClN}_{6} \mathrm{O}_{2}$ & $\mathrm{C}_{11} \mathrm{H}_{22} \mathrm{ClF}_{4} \mathrm{~N}_{3}$ \\
\hline & \multicolumn{2}{|c|}{$\mathrm{C}_{8} \mathrm{H}_{8} \mathrm{~N}_{2} \mathrm{~S}$} & \multicolumn{2}{|c|}{$\mathrm{C}_{11} \mathrm{H}_{11} \mathrm{~N}, \mathrm{O}_{2}$} & $\mathrm{C}_{22} \mathrm{H}_{18} \mathrm{~N}_{2} \mathrm{O}_{4}$ & $\mathrm{C}_{15} \mathrm{H}_{17} \mathrm{Cl}_{2} \mathrm{~N}_{3} \mathrm{O}_{2}$ \\
\hline & \multicolumn{2}{|c|}{$\mathrm{C}_{2} \mathrm{H}_{10} \mathrm{ClFO}$} & \multicolumn{2}{|c|}{$\mathrm{C}_{13} \mathrm{H}_{14} \mathrm{~F}_{3} \mathrm{~N}_{3} \mathrm{O}_{2}$} & $\mathrm{C}_{15} \mathrm{H}_{18} \mathrm{~N}_{8} \mathrm{O}_{2} \mathrm{~S}$ & $\mathrm{C}_{12} \mathrm{H}_{13} \mathrm{Cl}_{2} \mathrm{FN}_{3} \mathrm{O}_{3}$ \\
\hline & & & \multicolumn{2}{|c|}{$\mathrm{C}_{10} \mathrm{H}_{2} \mathrm{NO}_{5} \mathrm{~S}_{2}$} & $\mathrm{C}_{13} \mathrm{H}_{17} \mathrm{~F}_{3} \mathrm{~N}_{8} \mathrm{~S}$ & $\mathrm{C}_{10} \mathrm{H}_{10} \mathrm{Cl}_{2} \mathrm{~F}_{4} \mathrm{~N}_{3} \mathrm{O}$ \\
\hline & & & \multicolumn{2}{|c|}{$\mathrm{C}_{19} \mathrm{H}_{12} \mathrm{FN}_{3}$} & $\mathrm{C}_{1}, \mathrm{H}_{2}, \mathrm{ClN}_{2} \mathrm{OS}_{2}$ & $\mathrm{C}_{33} \mathrm{H}_{16} \mathrm{Cl}_{2} \mathrm{~F}_{3} \mathrm{~N}_{3}$ \\
\hline & & & \multicolumn{2}{|c|}{$\mathrm{C}_{10} \mathrm{H}_{99} \mathrm{~N}_{3} \mathrm{O}$} & $\mathrm{C}_{19} \mathrm{H}_{21} \mathrm{CIN}_{3} \mathrm{O}_{3}$ & $\mathrm{C}_{14} \mathrm{H}_{4}, \mathrm{Cl}_{2} \mathrm{~N}_{3} \mathrm{O}$ \\
\hline & & & \multicolumn{2}{|c|}{$\mathrm{C}_{10} \mathrm{H}_{9} \mathrm{~N}_{3} \mathrm{~S}$} & & $\mathrm{C}_{11} \mathrm{H}_{12} \mathrm{Cl}_{2} \mathrm{FN}_{3} \mathrm{O}_{2}$ \\
\hline & & & \multicolumn{2}{|c|}{$\mathrm{C}_{10} \mathrm{H}_{8} \mathrm{~N}_{4} \mathrm{~S}$} & & $\mathrm{C}_{2} \mathrm{H}_{1}, \mathrm{Cl}_{2} \mathrm{~F}_{2} \mathbf{N}_{3}$ \\
\hline & & & \multicolumn{2}{|c|}{$\mathrm{C}_{9} \mathrm{H}_{6} \mathrm{ClN}_{3} \mathrm{~S}$} & & \\
\hline & & & $C_{2} \mathrm{H}_{4} \mathbf{F}$ & $F_{s} N_{3}$ & & \\
\hline & & & $\mathrm{C}_{8} \mathrm{H}_{7} \mathrm{Br}$ & $\mathrm{r} N \mathrm{~N}_{2} \mathrm{~S}$ & & \\
\hline & & & $\mathrm{C}_{13} \mathrm{H}_{3,}, \mathrm{~V}$ & $\mathrm{~N}_{3} \mathrm{O}_{4}$ & & \\
\hline
\end{tabular}

\begin{tabular}{|c|c|c|c|c|c|}
\hline F1-3 & $\mathrm{C}_{9} \mathrm{H}_{2} \mathrm{~N}_{3} \mathrm{~S}$ & $\mathrm{C}_{3} \mathrm{H}_{2} \mathrm{~N}_{2} \mathrm{~S}$ & & & \\
\hline $\begin{array}{l}\text { Structures (In silicec } \\
\text { fragmentation) }\end{array}$ & 16 & 20 & & & \\
\hline In-house QSRR & 9 & 17 & & & \\
\hline 48-h LCs (mg/L) & 33.14 & 26.75 & & & \\
\hline $\begin{array}{l}\text { Commercial } \\
\text { Importance }\end{array}$ & 19062 & 873 & & & \\
\hline F1-4 & $\mathrm{C}_{16} \mathrm{H}_{13} \mathrm{~F}_{2} \mathrm{~N}_{3} \mathrm{O}$ & $\mathrm{C}_{10} \mathrm{H}_{19}, \mathrm{~N}_{3} \mathrm{O}$ & $\mathrm{C}_{10 \mathrm{H}} \mathrm{N}_{3} \mathrm{~S}$ & $\mathrm{C}_{10} \mathrm{H}_{8} \mathrm{~N}_{4} \mathrm{~S}$ & $\mathrm{C}_{8} \mathrm{H}_{7} \mathrm{BrN}_{2} \mathrm{~S}$ \\
\hline $\begin{array}{l}\text { Structures (In silicec } \\
\text { fragmentation) }\end{array}$ & 8 & 248 & 118 & 189 & 200 \\
\hline In-house QSRR & 2 & 8 & 58 & 55 & 61 \\
\hline 48-h LCss (mg/L) & 52.04 & 32.68 & 35.74 & 38.89 & 30.66 \\
\hline $\begin{array}{l}\text { Commercial } \\
\text { Importance }\end{array}$ & 38009 & 1 & 2 & 10 & 6 \\
\hline F1-5 & $\mathrm{C}_{16 \mathrm{H}} 4 \mathrm{NO}_{2}$ & $\mathrm{C}_{17} \mathrm{H}_{10} \mathrm{ClN}_{6} \mathrm{O}_{2}$ & & & \\
\hline $\begin{array}{l}\text { Structures (In silice } \\
\text { fragmentation) }\end{array}$ & 15 & 23 & & & \\
\hline In-house QSRR & 1 & 15 & & & \\
\hline 48-h LCs (mg/L) & 3.65 & 68.14 & & & \\
\hline $\begin{array}{l}\text { Commercial } \\
\text { Importance }\end{array}$ & 6 & 9 & & & \\
\hline F1-7 & $\mathrm{C}_{3} \mathrm{H}_{2} \mathrm{CbuN}_{3} \mathrm{O}_{2}$ & $\mathrm{C}_{2} \mathrm{H}_{2} \mathrm{CllN}_{3} \mathrm{O}$ & $\mathrm{CuH}_{4} \mathrm{ClN}_{3} \mathrm{O}$ & & \\
\hline $\begin{array}{l}\text { Structures (In silice } \\
\text { fragmentation) }\end{array}$ & 3 & 15 & 20 & & \\
\hline In-house QSRR & 1 & 4 & 4 & & \\
\hline $48-\mathrm{h} \mathrm{LC} C_{s 0}(\mathrm{mg} / \mathrm{L})$ & 4.34 & 3.12 & 6.70 & & \\
\hline $\begin{array}{l}\text { Commercinl } \\
\text { Importance }\end{array}$ & 57532 & 58202 & 43070 & & \\
\hline
\end{tabular}
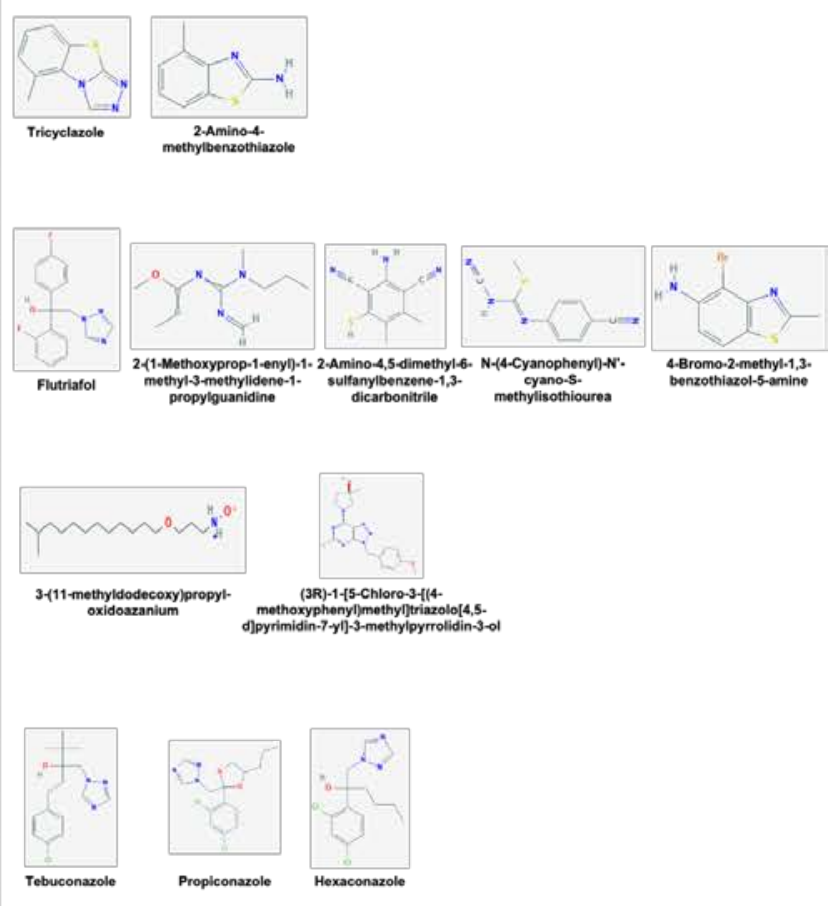
Figure S6 Removal efficiency (\%) of identified causative toxicants by conventional treatments in JT-CIP.

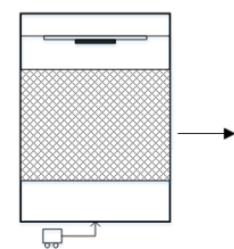

Microelectrolysis

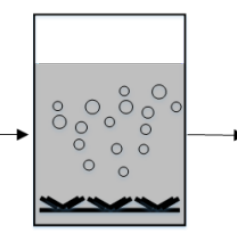

Fenton Oxidation

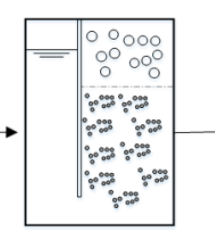

Anaerobic Tank

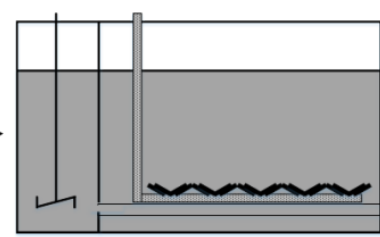

Nitrification + Denitrification Second Settling Pool

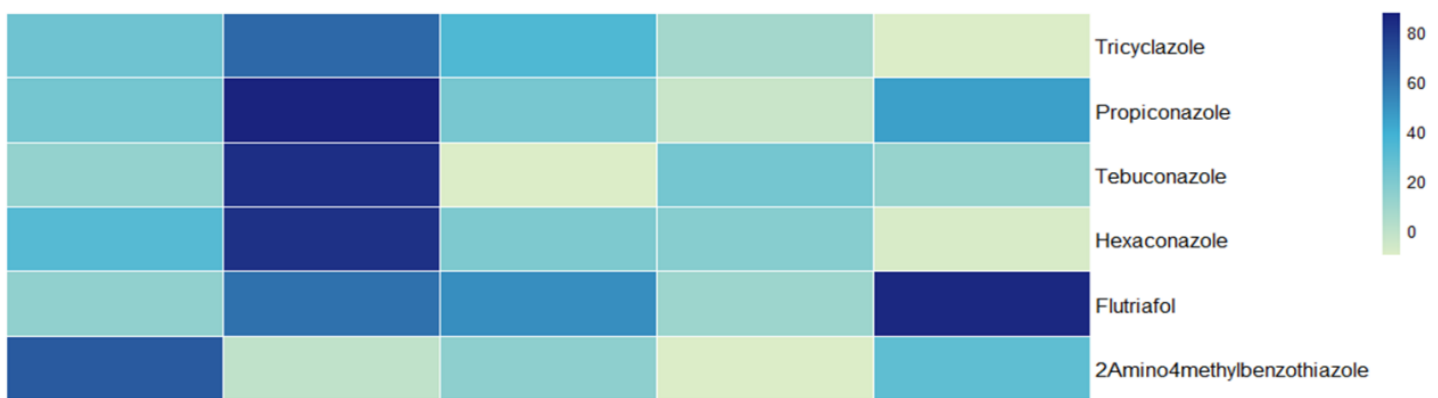


Table S1 Water quality parameters of wastewaters from different treatment

processes.

\begin{tabular}{ccccc}
\hline Treatment Processes & Temperature $/{ }^{\circ} \mathrm{C}$ & $\begin{array}{c}\text { Conductivity } \\
\left(\mu \mathrm{s} / \mathrm{cm}^{2}\right)\end{array}$ & $\mathrm{pH}$ & $\begin{array}{c}\text { Dissolved oxygen } \\
(\mathrm{mg} / \mathrm{L})\end{array}$ \\
\hline Influents & 23.28 & 32226 & 6.90 & 0.20 \\
Microelectrolysis & 21.97 & 26645 & 5.13 & 4.10 \\
Fenton Oxidation & 25.07 & 20844 & 3.01 & 6.10 \\
Anaerobic Tank & 24.74 & 6502 & 7.86 & 0.15 \\
Denitrification & 23.82 & 7058 & 8.11 & 8.06 \\
$\quad$ Nitrification+ & 26.27 & 7138 & 8.09 & 6.70 \\
Second settling pool & & 3793 & 7.88 & 8.05 \\
Effluents & 22.22 & & & \\
\hline
\end{tabular}


Table S2. List of artificial suspect compounds for evaluation of the sample preparation.

\begin{tabular}{|c|c|c|c|c|c|c|c|}
\hline Chemical Name & CAS No. & Formula & LogKow & Chemical Name & CAS No. & Formula & LogKow \\
\hline Imidacloprid & $138261-41-3$ & $\mathrm{C}_{9} \mathrm{H}_{10} \mathrm{ClN}_{5} \mathrm{O}_{2}$ & 0.56 & Pirimiphos-methyl & 29232-93-7 & $\mathrm{C}_{11} \mathrm{H}_{20} \mathrm{~N}_{3} \mathrm{O}_{3} \mathrm{PS}$ & 3.44 \\
\hline Fluometuron & $2164-17-2$ & $\mathrm{C}_{10} \mathrm{H}_{11} \mathrm{~F}_{3} \mathrm{~N}_{2} \mathrm{O}$ & 2.35 & Prosulfocrab & 52888-80-9 & $\mathrm{C}_{14} \mathrm{H}_{21} \mathrm{NOS}$ & 4.23 \\
\hline Buprofezin & 69327-76-0 & $\mathrm{C}_{16} \mathrm{H}_{23} \mathrm{~N}_{3} \mathrm{OS}$ & 4.30 & Terbutryn & $886-50-0$ & $\mathrm{C}_{10} \mathrm{H}_{19} \mathrm{~N}_{5} \mathrm{~S}$ & 3.77 \\
\hline Vitavax & $5234-68-4$ & $\mathrm{C}_{12} \mathrm{H}_{13} \mathrm{NO}_{2} \mathrm{~S}$ & 1.49 & Triadimefon & 43121-43-3 & $\mathrm{C}_{14} \mathrm{H}_{16} \mathrm{ClN}_{3} \mathrm{O}_{2}$ & 2.94 \\
\hline Cloquintocet-mexyl & $99607-70-2$ & $\mathrm{C}_{18} \mathrm{H}_{22} \mathrm{ClNO}_{3}$ & 5.28 & Cimetidine & 51481-61-9 & $\mathrm{C}_{10} \mathrm{H}_{16} \mathrm{~N}_{6} \mathrm{~S}$ & 0.57 \\
\hline Cyproconazole & $94361-06-5$ & $\mathrm{C}_{15} \mathrm{H}_{18} \mathrm{ClN} \mathrm{C}_{3} \mathrm{O}$ & 3.25 & Bensulfuron methyl & 83055-99-6 & $\mathrm{C}_{16} \mathrm{H}_{18} \mathrm{~N}_{4} \mathrm{O}_{7} \mathrm{~S}$ & 1.41 \\
\hline Diazinon & $333-41-5$ & $\mathrm{C}_{12} \mathrm{H}_{21} \mathrm{~N}_{2} \mathrm{O}_{3} \mathrm{PS}$ & 3.86 & Isoproturon & $34123-59-6$ & $\mathrm{C}_{12} \mathrm{H}_{18} \mathrm{~N}_{2} \mathrm{O}$ & 2.84 \\
\hline Difenoconazole & 119446-68-3 & $\mathrm{C}_{19} \mathrm{H}_{17} \mathrm{Cl}_{2} \mathrm{~N}_{3} \mathrm{O}_{3}$ & 5.20 & Pyrimethanil & 53112-28-0 & $\mathrm{C}_{12} \mathrm{H}_{13} \mathrm{~N}_{3}$ & 3.19 \\
\hline Diuron & $330-54-1$ & $\mathrm{C}_{9} \mathrm{H}_{10} \mathrm{Cl}_{2} \mathrm{~N}_{2} \mathrm{O}$ & 2.67 & Lidocaine & $137-58-6$ & $\mathrm{C}_{14} \mathrm{H}_{22} \mathrm{~N}_{2} \mathrm{O}$ & 1.66 \\
\hline Linuron & $330-55-2$ & $\mathrm{C}_{9} \mathrm{H}_{10} \mathrm{Cl}_{2} \mathrm{~N}_{2} \mathrm{O}_{2}$ & 2.91 & Nicotine & $54-11-5$ & $\mathrm{C}_{10} \mathrm{H}_{14} \mathrm{~N}_{2}$ & 1.00 \\
\hline Malathion & $121-75-5$ & $\mathrm{C}_{10} \mathrm{H}_{19} \mathrm{O}_{6} \mathrm{PS}_{2}$ & 2.29 & Sulpiride & 15676-16-1 & $\mathrm{C}_{15} \mathrm{H}_{23} \mathrm{~N}_{3} \mathrm{O}_{4} \mathrm{~S}$ & 0.65 \\
\hline Metazachlor & 67129-08-2 & $\mathrm{C}_{14} \mathrm{H}_{16} \mathrm{ClN} 3 \mathrm{O}$ & 2.38 & Telmisartan & $144701-48-4$ & $\mathrm{C}_{33} \mathrm{H}_{30} \mathrm{~N}_{4} \mathrm{O}_{2}$ & 8.42 \\
\hline Praziquantel & 55268-74-1 & $\mathrm{C}_{19} \mathrm{H}_{24} \mathrm{~N}_{2} \mathrm{O}_{2}$ & 2.42 & Terbutylazine & $5915-41-3$ & $\mathrm{C}_{9} \mathrm{H}_{16} \mathrm{ClN}_{5}$ & 3.27 \\
\hline Prometon & $1610-18-0$ & $\mathrm{C}_{10} \mathrm{H}_{19} \mathrm{~N}_{5} \mathrm{O}$ & 3.57 & Fluconazole & 86386-73-4 & $\mathrm{C}_{13} \mathrm{H}_{12} \mathrm{~F}_{2} \mathrm{~N}_{6} \mathrm{O}$ & 0.25 \\
\hline Propamocarb & $24579-73-5$ & $\mathrm{C}_{9} \mathrm{H}_{20} \mathrm{~N}_{2} \mathrm{O}_{2}$ & 1.13 & Irbesartan & 138402-11-6 & $\mathrm{C}_{25} \mathrm{H}_{28} \mathrm{~N}_{6} \mathrm{O}$ & 5.31 \\
\hline Thiabendazole & $148-79-8$ & $\mathrm{C}_{10} \mathrm{H}_{7} \mathrm{~N}_{3} \mathrm{~S}$ & 2.00 & Ornidazole & $16773-42-5$ & $\mathrm{C}_{7} \mathrm{H}_{10} \mathrm{ClN}_{3} \mathrm{O}_{3}$ & 0.67 \\
\hline Thiamethoxam & 153719-23-4 & $\mathrm{C}_{8} \mathrm{H}_{10} \mathrm{ClN}_{5} \mathrm{O}_{3} \mathrm{~S}$ & 0.80 & Melamine & $108-78-1$ & $\mathrm{C}_{3} \mathrm{H}_{6} \mathrm{~N}_{6}$ & -0.38 \\
\hline 2-Benzoylacetanilide & $103-84-4$ & $\mathrm{C}_{8} \mathrm{H}_{9} \mathrm{NO}$ & 1.10 & Erythromycin & $114-07-8$ & $\mathrm{C}_{37} \mathrm{H}_{67} \mathrm{NO}_{13}$ & 2.48 \\
\hline Triisopropanolamine & $122-20-3$ & $\mathrm{C}_{9} \mathrm{H}_{21} \mathrm{NO}_{3}$ & -1.22 & Venlafaxine & 93413-69-5 & $\mathrm{C}_{17} \mathrm{H}_{27} \mathrm{NO}_{2}$ & 3.28 \\
\hline Levamisole & 14769-73-4 & $\mathrm{C}_{11} \mathrm{H}_{12} \mathrm{~N}_{2} \mathrm{~S}$ & 2.87 & Nicosulfuron & 111991-09-4 & $\mathrm{C}_{15} \mathrm{H}_{18} \mathrm{~N}_{6} \mathrm{O}_{6} \mathrm{~S}$ & -1.15 \\
\hline Ketoconazole & $65277-42-1$ & $\mathrm{C}_{26} \mathrm{H}_{28} \mathrm{Cl}_{2} \mathrm{~N}_{4} \mathrm{O}_{4}$ & 4.45 & 2,4-Dichlorophenol & $120-83-2$ & $\mathrm{C}_{6} \mathrm{H}_{4} \mathrm{Cl}_{2} \mathrm{O}$ & 2.80 \\
\hline Dimethomorph & 110488-70-5 & $\mathrm{C}_{21} \mathrm{H}_{22} \mathrm{ClNO}_{4}$ & 2.36 & Chloramphenicol & $56-75-7$ & $\mathrm{C}_{11} \mathrm{H}_{12} \mathrm{Cl}_{2} \mathrm{~N}_{2} \mathrm{O}_{5}$ & 0.92 \\
\hline Imazalil & $35554-44-0$ & $\mathrm{C}_{14} \mathrm{H}_{14} \mathrm{Cl}_{2} \mathrm{~N}_{2} \mathrm{O}$ & 4.10 & N-Lauroylsarcosine & $97-78-9$ & $\mathrm{C}_{15} \mathrm{H}_{29} \mathrm{NO}_{3}$ & 4.10 \\
\hline
\end{tabular}




\begin{tabular}{|c|c|c|c|c|c|c|c|}
\hline Metalaxyl & $57837-19-1$ & $\mathrm{C}_{15} \mathrm{H}_{21} \mathrm{NO}_{4}$ & 1.70 & Fluroxyрyг & 69377-81-7 & $\mathrm{C}_{7} \mathrm{H}_{5} \mathrm{Cl}_{2} \mathrm{FN}_{2} \mathrm{O}_{3}$ & 1.17 \\
\hline Prochloraz & 67747-09-5 & $\mathrm{C}_{15} \mathrm{H}_{16} \mathrm{Cl}_{3} \mathrm{~N}_{3} \mathrm{O}_{2}$ & 4.13 & $\begin{array}{l}\text { 2-Methyl-4- } \\
\text { chlorophenoxyacetic acid }\end{array}$ & $94-74-6$ & $\mathrm{C}_{9} \mathrm{H}_{9} \mathrm{ClO}_{3}$ & 2.52 \\
\hline Propiconazole & $60207-90-1$ & $\mathrm{C}_{15} \mathrm{H}_{17} \mathrm{Cl}_{2} \mathrm{~N}_{3} \mathrm{O}_{2}$ & 4.13 & $\begin{array}{l}\text { 2-Amino-4,6- } \\
\text { dimethoxypyrimidine }\end{array}$ & $36315-01-2$ & $\mathrm{C}_{6} \mathrm{H}_{9} \mathrm{~N}_{3} \mathrm{O}_{2}$ & 0.95 \\
\hline Tebuconazole & $107534-96-3$ & $\mathrm{C}_{16} \mathrm{H}_{22} \mathrm{ClN}_{3} \mathrm{O}$ & 3.89 & Benzimidazole & $51-17-2$ & $\mathrm{C}_{7} \mathrm{H}_{6} \mathrm{~N}_{2}$ & 1.23 \\
\hline Atrazine & $1912-24-9$ & $\mathrm{C}_{8} \mathrm{H}_{14} \mathrm{ClN} 5$ & 2.82 & Imidacloprid-urea & 120868-66-8 & $\mathrm{C}_{9} \mathrm{H}_{10} \mathrm{ClN}_{3} \mathrm{O}$ & 0.65 \\
\hline Azoxystrobin & 131860-33-8 & $\mathrm{C}_{22} \mathrm{H}_{17} \mathrm{~N}_{3} \mathrm{O}_{5}$ & 1.58 & Atrazine-2-hydroxy & 2163-68-0 & $\mathrm{C}_{8} \mathrm{H}_{15} \mathrm{~N}_{5} \mathrm{O}$ & 2.09 \\
\hline Carbendazim & $10605-21-7$ & $\mathrm{C}_{9} \mathrm{H}_{9} \mathrm{~N}_{3} \mathrm{O}_{2}$ & 1.55 & Propazine-2-hydroxy & $7374-53-0$ & $\mathrm{C}_{9} \mathrm{H}_{17} \mathrm{~N}_{5} \mathrm{O}$ & 2.51 \\
\hline (S)-Metolachlor & $87392-12-9$ & $\mathrm{C}_{15} \mathrm{H}_{22} \mathrm{ClNO}_{2}$ & 3.24 & Clothianidin & 210880-92-5 & $\mathrm{C}_{6} \mathrm{H}_{8} \mathrm{ClN}_{5} \mathrm{O}_{2} \mathrm{~S}$ & 0.64 \\
\hline Prometryn & $7287-19-6$ & $\mathrm{C}_{10} \mathrm{H}_{19} \mathrm{~N}_{5} \mathrm{~S}$ & 3.73 & Cloquintocet & 88349-88-6 & $\mathrm{C}_{11} \mathrm{H}_{8} \mathrm{ClNO}_{3}$ & 2.12 \\
\hline Tricyclazole & $41814-78-2$ & $\mathrm{C}_{9} \mathrm{H}_{7} \mathrm{~N}_{3} \mathrm{~S}$ & 2.48 & 4,6-Dimethoxypyrimidine & $5270-94-0$ & $\mathrm{C}_{6} \mathrm{H}_{8} \mathrm{~N}_{2} \mathrm{O}_{2}$ & 1.01 \\
\hline Amantadine & $768-94-5$ & $\mathrm{C}_{10} \mathrm{H}_{17} \mathrm{~N}$ & 2.43 & 2,4,6-Trichlorophenol & 88-06-2 & $\mathrm{C}_{6} \mathrm{H}_{3} \mathrm{Cl}_{3} \mathrm{O}$ & 3.45 \\
\hline Flutriafol & 76674-21-0 & $\mathrm{C}_{16} \mathrm{H}_{13} \mathrm{~F}_{2} \mathrm{~N}_{3} \mathrm{O}$ & 2.52 & $\begin{array}{l}\text { 2,4-Dichlorophenoxyacetic } \\
\text { acid }\end{array}$ & 94-75-7 & $\mathrm{C}_{8} \mathrm{H}_{6} \mathrm{Cl}_{2} \mathrm{O}_{3}$ & 2.62 \\
\hline Acetamiprid & 135410-20-7 & $\mathrm{C}_{10} \mathrm{H}_{11} \mathrm{ClN}_{4}$ & 2.55 & Roxithromycin & 80214-83-1 & $\mathrm{C}_{41} \mathrm{H}_{76} \mathrm{~N}_{2} \mathrm{O}_{15}$ & 2.75 \\
\hline Clomazone & 81777-89-1 & $\mathrm{C}_{12} \mathrm{H}_{14} \mathrm{ClNO}_{2}$ & 2.86 & $\begin{array}{l}\text { Bis(4-fluorophenyl)- } \\
\text { methanone }\end{array}$ & $345-92-6$ & $\mathrm{C}_{13} \mathrm{H}_{8} \mathrm{~F}_{2} \mathrm{O}$ & 3.55 \\
\hline Isoprothiolane & 50512-35-1 & $\mathrm{C}_{12} \mathrm{H}_{18} \mathrm{O}_{4} \mathrm{~S}_{2}$ & 2.79 & & & & \\
\hline
\end{tabular}


Table S3 Collection times and logкоw ranges of preparative fractions.

\begin{tabular}{lll}
\hline Fraction Name & Collection Time & LogKow Range \\
\hline F1-1 & $0-5 \mathrm{~min}$ & $0.33-0.47$ \\
F1-2 & $5-10 \mathrm{~min}$ & $0.24-1.05$ \\
F1-3 & $10-15 \mathrm{~min}$ & $1.06-2.03$ \\
F1-4 & $15-20 \mathrm{~min}$ & $1.76-2.74$ \\
F1-5 & $20-26 \mathrm{~min}$ & $2.44-3.56$ \\
F1-6 & $26-30 \mathrm{~min}$ & $2.68-3.33$ \\
F1-7 & $30-38 \mathrm{~min}$ & $3.42-4.68$ \\
F1-8 & $38-52 \mathrm{~min}$ & $3.84-6.09$ \\
F1-9 & $52-60 \mathrm{~min}$ & $5.58-6.87$ \\
F1-10 & $60-70 \mathrm{~min}$ & $6.46-8.07$ \\
F1-11 & $70-80 \mathrm{~min}$ & $7.61-9.22$ \\
\hline
\end{tabular}


Table S4 Quantitation ions and limit of detection (LOD) values of causative toxicants.

\begin{tabular}{|c|c|c|c|c|}
\hline Chemical Name & $\begin{array}{l}\text { Quantitative } \\
\text { Ions }\end{array}$ & $\mathrm{CE}(\mathrm{eV})$ & $\mathrm{LOQ}(\mu \mathrm{g} / \mathrm{L})$ & $\mathrm{LOD}(\mu \mathrm{g} / \mathrm{L})$ \\
\hline \multirow{2}{*}{ Tricyclazole } & $189.9>163.1$ & 33 & \multirow{2}{*}{2} & \multirow{2}{*}{$<0.5$} \\
\hline & $189.9>136.1$ & 40 & & \\
\hline \multirow{2}{*}{ Flutriafol } & $301.9>123.0$ & 44 & \multirow{2}{*}{2} & \multirow{2}{*}{$<0.5$} \\
\hline & $301.9>70.1$ & 46 & & \\
\hline \multirow{2}{*}{$\begin{array}{l}\text { 2-Amino-4- } \\
\text { methylbenzothiazole }\end{array}$} & $164.9>123.1$ & 35 & \multirow{2}{*}{1} & \multirow{2}{*}{$<0.1$} \\
\hline & $164.9>79.1$ & 42 & & \\
\hline \multirow{2}{*}{ Tebuconazole } & $308.1>125.1$ & 55 & \multirow{2}{*}{2} & \multirow{2}{*}{$<0.5$} \\
\hline & $308.1>70.1$ & 51 & & \\
\hline \multirow{2}{*}{ Propiconazole } & $342.0>159.0$ & 41 & \multirow{2}{*}{2} & \multirow{2}{*}{$<0.5$} \\
\hline & $342.0>69.1$ & 42 & & \\
\hline \multirow{2}{*}{ Hexaconazole } & $314.0>158.9$ & 42 & \multirow{2}{*}{2} & \multirow{2}{*}{$<0.5$} \\
\hline & $314.0>70.1$ & 36 & & \\
\hline
\end{tabular}

Note: $\mathrm{CE}(\mathrm{eV})$ is the collision energy of individual quantitative ions. $\mathrm{DP}(\mathrm{V})$ is the declustering potential of individual quantitative ions. 
Table S6 Transformation products (TPs) of identified toxicants.

\begin{tabular}{|c|c|c|}
\hline Chemicals & SMILES of Transformation Products & $\begin{array}{l}48 \mathrm{~h} \mathrm{EC}_{50} \\
(\mathrm{mg} / \mathrm{L})\end{array}$ \\
\hline \multirow[t]{13}{*}{ Tricyclazole } & Cc1cccc2sc3nncn3c12 & $3.31 \times 10^{1}$ \\
\hline & $\mathrm{CC} 1=\mathrm{C} 2 \mathrm{C}(=\mathrm{CC}=\mathrm{C} 1) \mathrm{SC}(=\mathrm{N} 2) \mathrm{N}$ & $2.68 \times 10^{1}$ \\
\hline & $\mathrm{Cc} 1 \mathrm{cc}(\mathrm{O}) \mathrm{c}(\mathrm{O}) \mathrm{c} 2 \mathrm{sc} 3 \mathrm{nnn} n 3 \mathrm{c} 12$ & $2.35 \times 10^{2}$ \\
\hline & OCc1cccc2sc3nncn3c12 & $5.48 \times 10^{2}$ \\
\hline & $\mathrm{C} \| \mathrm{C}(=\mathrm{C} \| \mathrm{C}([\mathrm{O}-])=\mathrm{O}) \mathrm{c} 1 \mathrm{c}(\mathrm{sc} 2 \mathrm{nncn} 12) \mathrm{C}([\mathrm{O}-])=\mathrm{O}$ & $4.36 \times 10^{3}$ \\
\hline & $\mathrm{O}=\mathrm{Cc} 1 \mathrm{ccc} 2 \mathrm{sc} 3 \mathrm{nnn} n 3 \mathrm{c} 12$ & $1.70 \times 10^{2}$ \\
\hline & OCc1cc(O)c(O)c2sc3nncn3c12 & $3.66 \times 10^{3}$ \\
\hline & $\mathrm{CC}(\mathrm{O})(\mathrm{CC}([\mathrm{O}-])=\mathrm{O}) \mathrm{c} 1 \mathrm{c}(\mathrm{sc} 2 \mathrm{nnncn} 12) \mathrm{C}([\mathrm{O}-])=\mathrm{O}$ & $6.13 \times 10^{3}$ \\
\hline & $\mathrm{C} \| \mathrm{C}(=\mathrm{C} \| \mathrm{C}([\mathrm{O}-])=\mathrm{O}) \mathrm{c} 1 \mathrm{csc} 2 \mathrm{nncn} 12$ & $2.88 \times 10^{3}$ \\
\hline & $\mathrm{C} \backslash \mathrm{C}(=\mathrm{C} \backslash \backslash \mathrm{C}([\mathrm{O}-])=\mathrm{O}) \mathrm{c} 1 \mathrm{c}(\mathrm{C}([\mathrm{O}-])=\mathrm{O}) \mathrm{s}(=\mathrm{O}) \mathrm{c} 2 \mathrm{nncn} 12$ & $9.98 \times 10^{3}$ \\
\hline & {$[\mathrm{O}-] \mathrm{C}(=\mathrm{O}) \mathrm{c} 1 \mathrm{cccc} 2 \mathrm{sc} 3 n n c n 3 \mathrm{c} 12$} & $1.34 \times 10^{3}$ \\
\hline & Oc1cc $(\mathrm{C}=\mathrm{O}) \mathrm{c} 2 \mathrm{c}(\mathrm{sc} 3 \mathrm{nnn} \operatorname{con} 23) \mathrm{c} 1 \mathrm{O}$ & $1.14 \times 10^{3}$ \\
\hline & $\mathrm{OC} \| \mathrm{C}(=\mathrm{C} \| \mathrm{C}([\mathrm{O}-])=\mathrm{O}) \mathrm{c} 1 \mathrm{c}(\operatorname{sc} 2 \mathrm{nncn} 12) \mathrm{C}([\mathrm{O}-])=\mathrm{O}$ & $6.64 \times 10^{3}$ \\
\hline \multirow[t]{26}{*}{ Propiconazole } & CCCC1COC(Cn2cncn2)(O1)c1ccc(Cl)cc1Cl & $3.12 \times 10^{0}$ \\
\hline & $\operatorname{CCCC}(=0) \operatorname{COC}(O)(\operatorname{Cn} 1 \mathrm{cncn} 1) \operatorname{c1ccc}(\mathrm{Cl}) \mathrm{cc} 1 \mathrm{Cl}$ & $1.45 \times 10^{2}$ \\
\hline & CCCC1OC(Cn2cncn2)(OC1O)c1ccc(Cl)cc1Cl & $1.15 \times 10^{1}$ \\
\hline & $\operatorname{CCCC}(\mathrm{OC}(\mathrm{O})(\mathrm{Cn} 1 \mathrm{cncn} 1) \mathrm{c} 1 \mathrm{ccc}(\mathrm{Cl}) \mathrm{cc} 1 \mathrm{Cl}) \mathrm{C}=\mathrm{O}$ & $1.48 \times 10^{2}$ \\
\hline & $\operatorname{CCCC}(=0) \operatorname{CO}$ & $1.22 \times 10^{3}$ \\
\hline & $\operatorname{CCCC}(=0) C=0$ & $4.40 \times 10^{3}$ \\
\hline & $\operatorname{Clc1} 1 \mathrm{ccc}(\mathrm{C}(=\mathrm{O}) \mathrm{Cn} 2 \mathrm{cncn} 2) \mathrm{c}(\mathrm{Cl}) \mathrm{c} 1$ & $1.33 \times 10^{2}$ \\
\hline & Oc1c $(\mathrm{Cl}) \mathrm{cc}(\mathrm{Cl}) \mathrm{c}(\mathrm{C}(=\mathrm{O}) \mathrm{Cn} 2 \mathrm{cncn} 2) \mathrm{c} 1 \mathrm{O}$ & $2.09 \times 10^{2}$ \\
\hline & $\operatorname{CCCC}(\mathrm{OC}(\mathrm{O})(\mathrm{Cn} 1 \mathrm{cncn} 1) \mathrm{c} 1 \mathrm{ccc}(\mathrm{Cl}) \mathrm{cc} 1 \mathrm{Cl}) \mathrm{C}([\mathrm{O}-])=\mathrm{O}$ & $9.77 \times 10^{1}$ \\
\hline & $\operatorname{CCCC}(=0) C([O-])=O$ & $3.53 \times 10^{3}$ \\
\hline & $\operatorname{CCCC}(\mathrm{O}) \mathrm{C}=\mathrm{O}$ & $2.68 \times 10^{3}$ \\
\hline & $\operatorname{CCCC}(O) C([O-])=O$ & $1.18 \times 10^{4}$ \\
\hline & $\operatorname{CCCC}([\mathrm{O}-])=\mathrm{O}$ & $2.20 \times 10^{3}$ \\
\hline & $\operatorname{OCCCC}(=0) C([O-])=0$ & $6.28 \times 10^{4}$ \\
\hline & {$[\mathrm{O}-] \mathrm{C}(=\mathrm{O}) \mathrm{C}(=\mathrm{O}) \mathrm{C}(\mathrm{Cl}) \backslash \mathrm{C}=\mathrm{C} \backslash \mathrm{Cl}$} & $1.70 \times 10^{4}$ \\
\hline & {$[\mathrm{O}-] \mathrm{C}(=\mathrm{O}) \mathrm{C}(=\mathrm{O}) \mathrm{CCl}$} & $1.46 \times 10^{4}$ \\
\hline & {$[\mathrm{O}-] \mathrm{C}(=\mathrm{O}) \mathrm{C}(=\mathrm{O}) \mathrm{Cn} 1 \mathrm{cncn} 1$} & $1.75 \times 10^{5}$ \\
\hline & {$[\mathrm{O}-] \mathrm{C}(=\mathrm{O}) \mathrm{Cn} 1 \mathrm{cncn} 1$} & $4.97 \times 10^{4}$ \\
\hline & {$[\mathrm{O}-] \mathrm{C}(=\mathrm{O}) \mathrm{C}(=\mathrm{O}) \mathrm{CCC}=\mathrm{O}$} & $5.48 \times 10^{4}$ \\
\hline & $\operatorname{OCCCC}([0-])=0$ & $4.07 \times 10^{4}$ \\
\hline & $\mathrm{OC}(\mathrm{C}(\mathrm{Cl}) \mathrm{C}([\mathrm{O}-])=\mathrm{O}) \mathrm{C}(\| \mathrm{Cl})=\mathrm{C} \| \mathrm{C}(=\mathrm{O}) \mathrm{Cn} 1 \mathrm{cncn} 1$ & $1.36 \times 10^{6}$ \\
\hline & $\mathrm{OC}(\mathrm{Cl})(\mathrm{CC}(=\mathrm{O}) \mathrm{Cn} 1 \mathrm{cncn} 1) \backslash \mathrm{C}=\mathrm{C}(\backslash \mathrm{Cl}) \mathrm{C}([\mathrm{O}-])=\mathrm{O}$ & $2.59 \times 10^{4}$ \\
\hline & {$[\mathrm{O}-] \mathrm{C}(=\mathrm{O}) \mathrm{CCC}([\mathrm{O}-])=\mathrm{O}$} & $9.06 \times 10^{4}$ \\
\hline & {$[\mathrm{O}-] \mathrm{C}(=\mathrm{O}) \mathrm{CC}(=\mathrm{O}) \mathrm{Cn} 1 \mathrm{cncn} 1$} & $1.18 \times 10^{5}$ \\
\hline & $\mathrm{OCC}(=\mathrm{O}) \mathrm{C}([\mathrm{O}-])=\mathrm{O}$ & $3.13 \times 10^{5}$ \\
\hline & {$[\mathrm{O}-] \mathrm{C}(=\mathrm{O}) \mathrm{CCl}$} & $9.37 \times 10^{3}$ \\
\hline
\end{tabular}


$\mathrm{CC}(\mathrm{C})(\mathrm{C}) \mathrm{C}(\mathrm{O})(\mathrm{Cn} 1 \mathrm{cncn} 1) \mathrm{C}(=\mathrm{O}) \mathrm{Cc} 1 \mathrm{ccc}(\mathrm{Cl}) \mathrm{cc} 1$

$7.23 \times 10^{2}$

Clc1 $\operatorname{ccc}(\operatorname{CCC}(=)) \operatorname{Cn} 2 \mathrm{cncn} 2) \mathrm{cc} 1$

$2.47 \times 10^{2}$

$\mathrm{OC}(\mathrm{Cc} 1 \mathrm{ccc}(\mathrm{Cl}) \mathrm{cc} 1) \mathrm{C}(=\mathrm{O}) \mathrm{Cn} 1 \mathrm{cncn} 1$

$8.18 \times 10^{2}$

$\mathrm{CC}(\mathrm{C}) \mathrm{C}([\mathrm{O}-])=\mathrm{O}$

$2.53 \times 10^{3}$

$\mathrm{CC}(\mathrm{C})(\mathrm{C}=\mathrm{O}) \mathrm{C}(\mathrm{O})(\mathrm{Cn} 1 \mathrm{cncn} 1) \mathrm{C}(=\mathrm{O}) \mathrm{C}(=\mathrm{O}) \mathrm{c} 1 \mathrm{ccc}(\mathrm{Cl}) \mathrm{cc} 1$

$6.16 \times 10^{3}$

$\mathrm{CC}(\mathrm{C})(\mathrm{C}) \mathrm{C}(=\mathrm{O}) \mathrm{Cn} 1 \mathrm{cncn} 1$

$2.48 \times 10^{3}$

$\mathrm{CC}(\mathrm{C})(\mathrm{CO}) \mathrm{C}(=\mathrm{O}) \mathrm{Cn} 1 \mathrm{cncn} 1$

$3.95 \times 10^{4}$

$\mathrm{Clc1}$ ccc $(\operatorname{cc} 1) \mathrm{C}(=\mathrm{O}) \mathrm{C}=\mathrm{O}$

$1.57 \times 10^{2}$

[O-]C $(=\mathrm{O}) \mathrm{C}(=\mathrm{O}) \mathrm{c} 1 \mathrm{ccc}(\mathrm{Cl}) \mathrm{cc} 1$

$1.07 \times 10^{2}$

$\mathrm{CC}(\mathrm{C})(\mathrm{C}([\mathrm{O}-])=\mathrm{O}) \mathrm{C}(\mathrm{O})(\mathrm{Cn} 1 \mathrm{cncn} 1) \mathrm{C}(=\mathrm{O}) \mathrm{C}(\mathrm{O}) \operatorname{c1ccc}(\mathrm{C}$ l)cc1

$3.95 \times 10^{2}$

$\mathrm{CC}(\mathrm{CO})(\mathrm{C}([\mathrm{O}-])=\mathrm{O}) \mathrm{C}(\mathrm{O})(\mathrm{Cn} 1 \mathrm{cncn} 1) \mathrm{C}(=\mathrm{O}) \mathrm{Cc} 1 \mathrm{ccc}(\mathrm{Cl})$ cc1

$2.72 \times 10^{3}$

Clc1ccc $(\mathrm{CC}(=\mathrm{O}) \mathrm{C}(=\mathrm{O}) \mathrm{Cn} 2 \mathrm{cncn} 2) \mathrm{cc} 1$

$4.80 \times 10^{3}$

$\mathrm{CC}(\mathrm{C}([\mathrm{O}-])=\mathrm{O})(\mathrm{C}([\mathrm{O}-])=\mathrm{O}) \mathrm{C}(\mathrm{O})(\mathrm{CCc} 1 \mathrm{ccc}(\mathrm{Cl}) \mathrm{cc} 1) \mathrm{Cn} 1$ encn1

$1.39 \times 10^{3}$

$\mathrm{CC}(\mathrm{CO}) \mathrm{C}([\mathrm{O}-])=\mathrm{O}$

$4.68 \times 10^{4}$

$\mathrm{CC}(\mathrm{C}=\mathrm{O}) \mathrm{C}([\mathrm{O}-])=\mathrm{O}$

$4.33 \times 10^{3}$

Hexaconazole

CCCCC(O)(Cn1cncn1)c1ccc(Cl)cc1Cl

$6.70 \times 10^{0}$

$\operatorname{CCCC}(\mathrm{O}) \mathrm{C}(\mathrm{O})(\mathrm{Cn} 1 \mathrm{cncn} 1) \mathrm{c} 1 \mathrm{ccc}(\mathrm{Cl}) \mathrm{cc} 1 \mathrm{Cl}$

$5.57 \times 10^{1}$

OCCCCC(O)(Cn1cncn1)c1ccc(Cl)cc1Cl

$4.87 \times 10^{1}$

$\operatorname{CCCC}(=0) \mathrm{C}(\mathrm{O})(\mathrm{Cn} 1 \mathrm{cncn} 1) \mathrm{c} 1 \mathrm{ccc}(\mathrm{Cl}) \mathrm{cc} 1 \mathrm{Cl}$

$1.41 \times 10^{1}$

OC(CCCC=O)(Cn1cncn1)c1ccc(Cl)cc1Cl

$1.06 \times 10^{2}$

$\mathrm{CCC}(\mathrm{O}) \mathrm{C}(=\mathrm{O}) \mathrm{C}(\mathrm{O})(\mathrm{Cn} 1 \mathrm{cncn} 1) \mathrm{c1} \operatorname{ccc}(\mathrm{Cl}) \mathrm{cc} 1 \mathrm{Cl}$

$1.48 \times 10^{1}$

OCCCC $(=\mathrm{O}) \mathrm{C}(\mathrm{O})(\mathrm{Cn} 1 \mathrm{cncn} 1) \mathrm{c} 1 \mathrm{ccc}(\mathrm{Cl}) \mathrm{cc} 1 \mathrm{Cl}$

$1.02 \times 10^{2}$

$\mathrm{OC}(\mathrm{CCCC}([\mathrm{O}-])=\mathrm{O})(\mathrm{Cn} 1 \mathrm{cncn} 1) \mathrm{c} 1 \mathrm{ccc}(\mathrm{Cl}) \mathrm{cc} 1 \mathrm{Cl}$

$7.03 \times 10^{1}$

$\operatorname{CCC}(=0) \mathrm{C}(=\mathrm{O}) \mathrm{C}(\mathrm{O})(\mathrm{Cn} 1 \mathrm{cncn} 1) \mathrm{c} 1 \mathrm{ccc}(\mathrm{Cl}) \mathrm{cc} 1 \mathrm{Cl}$

$5.67 \times 10^{3}$

OC(Cn1cncn1)(C(=O)CCC=O)c1ccc(Cl)cc1Cl

$2.23 \times 10^{2}$ 


\begin{tabular}{|c|c|c|}
\hline & $\mathrm{OC}(\mathrm{CC}([\mathrm{O}-])=\mathrm{O})(\mathrm{Cn} 1 \mathrm{cncn} 1) \mathrm{c} 1 \mathrm{ccc}(\mathrm{Cl}) \mathrm{cc} 1 \mathrm{Cl}$ & $3.89 \times 10^{2}$ \\
\hline & $\mathrm{CC}(\mathrm{O}) \mathrm{C}(=\mathrm{O}) \mathrm{C}(=\mathrm{O}) \mathrm{C}(\mathrm{O})(\mathrm{Cn} 1 \mathrm{cncn} 1) \mathrm{c} 1 \mathrm{ccc}(\mathrm{Cl}) \mathrm{cc} 1 \mathrm{Cl}$ & $4.70 \times 10^{4}$ \\
\hline & $\operatorname{OCCC}(=\mathrm{O}) \mathrm{C}(=\mathrm{O}) \mathrm{C}(\mathrm{O})(\mathrm{Cn} 1 \mathrm{cncn} 1) \mathrm{c} 1 \mathrm{ccc}(\mathrm{Cl}) \mathrm{cc} 1 \mathrm{Cl}$ & $4.11 \times 10^{4}$ \\
\hline & $\mathrm{OC}(\mathrm{Cn} 1 \mathrm{cncn} 1)(\mathrm{C}(=\mathrm{O}) \mathrm{CCC}([\mathrm{O}-])=\mathrm{O}) \mathrm{c} 1 \mathrm{ccc}(\mathrm{Cl}) \mathrm{cc} 1 \mathrm{Cl}$ & $1.47 \times 10^{2}$ \\
\hline & $\operatorname{Clc} 1 \mathrm{ccc}(\mathrm{C}(=\mathrm{O}) \mathrm{Cn} 2 \mathrm{cncn} 2) \mathrm{c}(\mathrm{Cl}) \mathrm{c} 1$ & $1.33 \times 10^{2}$ \\
\hline & $\mathrm{OC}(\mathrm{C}([\mathrm{O}-])=\mathrm{O}) \mathrm{C}(\mathrm{O})(\mathrm{Cn} 1 \mathrm{cncn} 1) \mathrm{c} 1 \mathrm{ccc}(\mathrm{Cl}) \mathrm{cc} 1 \mathrm{Cl}$ & $2.04 \times 10^{2}$ \\
\hline & $\mathrm{CC}(=\mathrm{O}) \mathrm{C}(=\mathrm{O}) \mathrm{C}(=\mathrm{O}) \mathrm{C}(\mathrm{O})(\mathrm{Cn} 1 \mathrm{cncn} 1) \mathrm{c} 1 \mathrm{ccc}(\mathrm{Cl}) \mathrm{cc} 1 \mathrm{Cl}$ & $4.03 \times 10^{3}$ \\
\hline & $\mathrm{OC}(\mathrm{Cn} 1 \mathrm{cncn} 1)(\mathrm{C}(=\mathrm{O}) \mathrm{C}(=\mathrm{O}) \mathrm{CC}=\mathrm{O}) \mathrm{c} 1 \mathrm{ccc}(\mathrm{Cl}) \mathrm{cc} 1 \mathrm{Cl}$ & $8.98 \times 10^{4}$ \\
\hline & Oc1c $(\mathrm{Cl}) \mathrm{cc}(\mathrm{Cl}) \mathrm{c}(\mathrm{C}(=\mathrm{O}) \mathrm{Cn} 2 \mathrm{cncn} 2) \mathrm{c} 1 \mathrm{O}$ & $2.09 \times 10^{2}$ \\
\hline & $\operatorname{CCC}(=\mathrm{O}) \mathrm{C}=\mathrm{O}$ & $9.28 \times 10^{3}$ \\
\hline & $\operatorname{CCC}(=0) C([O-])=O$ & $7.80 \times 10^{3}$ \\
\hline & $\mathrm{OC}(\mathrm{CC}([\mathrm{O}-])=\mathrm{O}) \mathrm{C}(=\mathrm{O}) \mathrm{C}(\mathrm{O})(\mathrm{Cn} 1 \mathrm{cncn} 1) \mathrm{c} 1 \mathrm{ccc}(\mathrm{Cl}) \mathrm{cc} 1 \mathrm{C}$ & $1.54 \times 10^{2}$ \\
\hline & $\mathrm{OC}(\mathrm{CC}(=\mathrm{O}) \mathrm{C}(\mathrm{O})(\mathrm{Cn} 1 \mathrm{cncn} 1) \operatorname{c1ccc}(\mathrm{Cl}) \operatorname{cc} 1 \mathrm{Cl}) \mathrm{C}([\mathrm{O}-])=$ & \\
\hline & $\mathrm{O}$ & $6.88 \times 10^{2}$ \\
\hline & $\mathrm{OC}(\mathrm{Cn} 1 \mathrm{cncn} 1)(\mathrm{C}(=\mathrm{O}) \mathrm{C}([\mathrm{O}-])=\mathrm{O}) \mathrm{c} 1 \mathrm{ccc}(\mathrm{Cl}) \mathrm{cc} 1 \mathrm{Cl}$ & $1.13 \times 10^{4}$ \\
\hline & $\mathrm{CC}(\mathrm{O}) \mathrm{C}(=\mathrm{O}) \mathrm{C}=\mathrm{O}$ & $1.83 \times 10^{5}$ \\
\hline & $\operatorname{OCCC}(=0) C=O$ & $1.60 \times 10^{5}$ \\
\hline Flutriafol & OC(Cn1cncn1)(c1ccc(F)cc1)c1ccccc1F & $5.20 \times 10^{1}$ \\
\hline & Oc1c(O)c(ccc1F)C(O)(Cn1cncn1)c1ccccc1F & $3.33 \times 10^{2}$ \\
\hline & Oc1ccc(F)c(c1O)C(O)(Cn1cncn1)c1ccc(F)cc1 & $3.33 \times 10^{2}$ \\
\hline & Oc1cc(F)c(cc1O)C(O)(Cn1cncn1)c1ccc(F)cc1 & $3.33 \times 10^{2}$ \\
\hline & Oc1ccc(c(F)c1O)C(O)(Cn1cncn1)c1ccc(F)cc1 & $3.33 \times 10^{2}$ \\
\hline & $\mathrm{OC}(\mathrm{Cn} 1 \mathrm{cncn} 1)(\mathrm{C}(=\mathrm{C} \backslash \mathrm{C}=\mathrm{C}(\backslash \mathrm{F}) \mathrm{C}([\mathrm{O}-])=\mathrm{O}) \backslash \mathrm{C}([\mathrm{O}-])=\mathrm{O}$ & $4.55 \times 10^{3}$ \\
\hline & )c1ccccc1F & \\
\hline & $\begin{array}{l}\mathrm{OC}(\mathrm{Cn} 1 \mathrm{cncn} 1)(\mathrm{C}(\mathrm{C}(\mathrm{F})=\mathrm{C}) \mathrm{C}(=\mathrm{O}) \mathrm{C}([\mathrm{O}-])=\mathrm{O}) \mathrm{c} 1 \mathrm{ccc}(\mathrm{F}) \mathrm{cc} \\
1\end{array}$ & $7.61 \times 10^{3}$ \\
\hline & $\begin{array}{l}\mathrm{OC}(\mathrm{Cn} 1 \mathrm{cncn} 1)(\mathrm{C}(=\mathrm{C}) \mathrm{C}(\mathrm{F}) \mathrm{C}(=\mathrm{O}) \mathrm{C}([\mathrm{O}-])=\mathrm{O}) \mathrm{c} 1 \mathrm{ccc}(\mathrm{F}) \mathrm{cc} \\
1\end{array}$ & $8.45 \times 10^{3}$ \\
\hline & {$[\mathrm{O}-] \mathrm{C}(=\mathrm{O}) \mathrm{C}(=\mathrm{O}) \mathrm{C}(\mathrm{F}) \mathrm{C}=\mathrm{C}$} & $6.65 \times 10^{3}$ \\
\hline & $\mathrm{OC}(\mathrm{Cn} 1 \mathrm{cncn} 1)(\mathrm{C}([\mathrm{O}-])=\mathrm{O}) \mathrm{c} 1 \mathrm{ccccc} 1 \mathrm{~F}$ & $4.95 \times 10^{3}$ \\
\hline & {$[\mathrm{O}-] \mathrm{C}(=\mathrm{O}) \mathrm{C}(=\mathrm{O}) \mathrm{C} \backslash \mathrm{C}=\mathrm{C} \backslash \mathrm{F}$} & $6.03 \times 10^{3}$ \\
\hline & $\mathrm{OC}(\mathrm{Cn} 1 \mathrm{cncn} 1)(\mathrm{C}([\mathrm{O}-])=\mathrm{O}) \mathrm{c} 1 \mathrm{ccc}(\mathrm{F}) \mathrm{cc} 1$ & $4.95 \times 10^{3}$ \\
\hline $\begin{array}{l}\text { (3R)-1-[5-Chloro-3- } \\
\text { [(4- }\end{array}$ & & \\
\hline methoxyphenyl)methy & COc1ccc(Cn2nnc3c(NCC[C@@](C)(O)C=O)nc(Cl)nc & $9.75 \times 10^{2}$ \\
\hline & & \\
\hline & $\mathrm{c} 12$ & $1.83 \times 10^{2}$ \\
\hline & COc1ccc(Cn2nnc3c(NC[C@](C)(O)CC([O-])=O)nc(Cl & $7.54 \times 10^{3}$ \\
\hline & )nc23)cc1 & \\
\hline
\end{tabular}


COc1ccc(Cn2nnc3c(NCC[C@@](C)(O)C([O-])=O)nc( Cl)nc23)cc1

C[C@](O)(CNc1nc(Cl)nc2n(Cc3ccc(O)cc3)nnc12)CC= $\mathrm{O}$

C[C@@](O)(CCNc1nc(Cl)nc2n(Cc3ccc(O)cc3)nnc12) $\mathrm{C}=\mathrm{O}$

C[C@@]1(O)CCN(C1)c1nc(Cl)nc2n(Cc3ccc(O)c(O)c3 )nnc12

COc1ccc(Cn2nnc3c(N)nc(Cl)nc23)cc1

C[C@@](O) $(\mathrm{CC}([\mathrm{O}-])=\mathrm{O}) \mathrm{C}=\mathrm{O}$

$\mathrm{C}[\mathrm{C} @](\mathrm{O})(\mathrm{CNc} 1 \mathrm{nc}(\mathrm{Cl}) \mathrm{nc} 2 \mathrm{n}(\mathrm{Cc} 3 \mathrm{ccc}(\mathrm{O}) \mathrm{cc} 3) \mathrm{nnc} 12) \mathrm{CC}($ $[\mathrm{O}-])=\mathrm{O}$

COc1ccc $(\mathrm{Cn} 2 \mathrm{nnnc} 3 \mathrm{c}(\mathrm{NCC}(\mathrm{C})=\mathrm{O}) \mathrm{nc}(\mathrm{Cl}) \mathrm{nc} 23) \mathrm{cc} 1$

COc1ccc $(\mathrm{Cn} 2 \mathrm{nnc} 3 \mathrm{c}(\mathrm{NCC}(\mathrm{C})=\mathrm{O}) \mathrm{nc}(\mathrm{Cl}) \mathrm{nc} 23) \mathrm{cc} 1$

$\mathrm{C}[\mathrm{C} @ @](\mathrm{O})(\mathrm{CC}=\mathrm{O}) \mathrm{C}([\mathrm{O}-])=\mathrm{O}$

C[C@@](O)(CCNc1nc(Cl)nc2n(Cc3ccc(O)cc3)nnc12)

$\mathrm{C}([\mathrm{O}-])=\mathrm{O}$

$\mathrm{C}[\mathrm{C} @](\mathrm{O})(\mathrm{CNc} 1 \mathrm{nc}(\mathrm{Cl}) \mathrm{nc} 2 \mathrm{n}(\mathrm{Cc} 3 \mathrm{ccc}(\mathrm{O}) \mathrm{c}(\mathrm{O}) \mathrm{c} 3) \mathrm{nnc12})$

$\mathrm{CC}=\mathrm{O}$

C[C@@](O)(CCNc1nc(Cl)nc2n(Cc3ccc(O)c(O)c3)nnc 12) $\mathrm{C}=\mathrm{O}$

C[C@@](O)(CCNc1nc(Cl)nc2n(Cc3ccc(O)c(O)c3)nnc 12) $\mathrm{C}=\mathrm{O}$

C[C@@]1(O)CCN(C1)c1nc(Cl)nc2n(ClC $(\backslash \mathrm{C}=\mathrm{C} / \mathrm{C}([\mathrm{O}-]$ )$=\mathrm{O})=\mathrm{ClC}([\mathrm{O}-])=\mathrm{O}) \mathrm{nnc} 12$

C[C@@]1(O)CCN(C1)c1nc(Cl)nc2n(CC $(=\mathrm{C}) \mathrm{CC}(=\mathrm{O}) \mathrm{C}$ $([\mathrm{O}-])=\mathrm{O}) \mathrm{nnc} 12$

C[C@@]1(O)CCN(C1)c1nc(Cl)nc2n(ClC=ClCC $(=O) C$ $([\mathrm{O}-])=\mathrm{O}) \mathrm{nnc} 12$

2-(1-Methoxyprop-1-

enyl)-1-methyl-3-

methylidene-1-

propylguanidine

$\mathrm{COC}(\mathrm{N})=\mathrm{CC}$
$4.27 \times 10^{3}$

$2.62 \times 10^{3}$

$2.62 \times 10^{3}$

$4.59 \times 10^{2}$

$2.64 \times 10^{2}$

$3.56 \times 10^{4}$

$3.13 \times 10^{3}$

$2.96 \times 10^{1}$

$2.96 \times 10^{1}$

$2.01 \times 10^{4}$

$1.15 \times 10^{4}$

$6.57 \times 10^{3}$

$6.57 \times 10^{3}$

$6.57 \times 10^{3}$

$5.61 \times 10^{4}$

$7.47 \times 10^{4}$

$9.55 \times 10^{4}$

$4.33 \times 10^{3}$

CCC $(\mathrm{N}) \mathrm{OC}$

$1.39 \times 10^{3}$

$\operatorname{CCCN}(\mathrm{C}) \mathrm{C}(=\mathrm{O}) \mathrm{N}=\mathrm{C}$

$9.86 \times 10^{2}$

$\mathrm{COC}(\mathrm{NC}(=\mathrm{O}) \mathrm{N}=\mathrm{C})=\mathrm{CC}$

$8.71 \times 10^{3}$

$\mathrm{CCC}(\mathrm{NC}(=\mathrm{O}) \mathrm{N}=\mathrm{C}) \mathrm{OC}$

$2.77 \times 10^{3}$

$\mathrm{CCCNC}$

$2.86 \times 10^{2}$

$\mathrm{CC}=\mathrm{C}(\mathrm{N}) \mathrm{O}$

$5.01 \times 10^{3}$

$\operatorname{CCC}(\mathrm{N})=\mathrm{O}$

$4.28 \times 10^{3}$

$\mathrm{CCC}(=\mathrm{O}) \mathrm{OC}$

$3.12 \times 10^{2}$

$\operatorname{CCCN}(\mathrm{C}) \mathrm{C}([\mathrm{O}-])=\mathrm{O}$

$3.27 \times 10^{3}$

$\mathrm{CC}(\mathrm{O}) \mathrm{CN}(\mathrm{C}) \mathrm{C}([\mathrm{O}-])=\mathrm{O}$

$6.68 \times 10^{4}$

$\mathrm{CN}(\mathrm{CCCO}) \mathrm{C}([\mathrm{O}-])=\mathrm{O}$
$5.82 \times 10^{4}$ 


\begin{tabular}{|c|c|}
\hline $\mathrm{CNC}(=\mathrm{O}) \mathrm{N}=\mathrm{C}$ & $5.86 \times 10^{3}$ \\
\hline $\mathrm{CCC}=\mathrm{O}$ & $5.51 \times 10^{2}$ \\
\hline $\operatorname{CCC}([\mathrm{O}-])=\mathrm{O}$ & $4.65 \times 10^{3}$ \\
\hline $\mathrm{CC}=\mathrm{C}(\mathrm{O}) \mathrm{NC}(=\mathrm{O}) \mathrm{N}=\mathrm{C}$ & $1.08 \times 10^{4}$ \\
\hline $\operatorname{CCC}(=\mathrm{O}) \mathrm{NC}(=\mathrm{O}) \mathrm{N}=\mathrm{C}$ & $6.36 \times 10^{4}$ \\
\hline CCCN & $5.42 \times 10^{2}$ \\
\hline $\mathrm{CCC}(\mathrm{N}) \mathrm{O}$ & $1.15 \times 10^{4}$ \\
\hline $\operatorname{CCC}(\mathrm{O}) \mathrm{NC}(=\mathrm{O}) \mathrm{N}=\mathrm{C}$ & $2.45 \times 10^{4}$ \\
\hline $\operatorname{CCCN}(\mathrm{C}) \mathrm{C}(\mathrm{N}=\mathrm{C})=\mathrm{NC}(\mathrm{OC})=\mathrm{CC}$ & $3.27 \times 10^{1}$ \\
\hline $\operatorname{COC}(\mathrm{N})=\mathrm{CC}$ & $4.33 \times 10^{3}$ \\
\hline $\mathrm{CCC}(\mathrm{N}) \mathrm{OC}$ & $1.39 \times 10^{3}$ \\
\hline $\operatorname{CCCN}(\mathrm{C}) \mathrm{C}(=\mathrm{O}) \mathrm{N}=\mathrm{C}$ & $9.86 \times 10^{2}$ \\
\hline $\operatorname{COC}(\mathrm{NC}(=\mathrm{O}) \mathrm{N}=\mathrm{C})=\mathrm{CC}$ & $8.71 \times 10^{3}$ \\
\hline $\operatorname{CCC}(\mathrm{NC}(=\mathrm{O}) \mathrm{N}=\mathrm{C}) \mathrm{OC}$ & $2.77 \times 10^{3}$ \\
\hline CCCNC & $2.86 \times 10^{2}$ \\
\hline $\mathrm{CC}=\mathrm{C}(\mathrm{N}) \mathrm{O}$ & $5.01 \times 10^{3}$ \\
\hline $\operatorname{CCC}(\mathrm{N})=\mathrm{O}$ & $4.28 \times 10^{3}$ \\
\hline $\operatorname{CCC}(=0) O C$ & $3.12 \times 10^{2}$ \\
\hline $\operatorname{CCCN}(\mathrm{C}) \mathrm{C}([\mathrm{O}-])=\mathrm{O}$ & $3.27 \times 10^{3}$ \\
\hline $\mathrm{CC}(\mathrm{O}) \mathrm{CN}(\mathrm{C}) \mathrm{C}([\mathrm{O}-])=\mathrm{O}$ & $6.68 \times 10^{4}$ \\
\hline $\mathrm{CN}(\mathrm{CCCO}) \mathrm{C}([\mathrm{O}-])=\mathrm{O}$ & $5.82 \times 10^{4}$ \\
\hline $\mathrm{CNC}(=\mathrm{O}) \mathrm{N}=\mathrm{C}$ & $5.86 \times 10^{3}$ \\
\hline $\mathrm{CCC}=\mathrm{O}$ & $5.51 \times 10^{2}$ \\
\hline $\operatorname{CCC}([\mathrm{O}-])=\mathrm{O}$ & $4.65 \times 10^{3}$ \\
\hline $\mathrm{CC}=\mathrm{C}(\mathrm{O}) \mathrm{NC}(=\mathrm{O}) \mathrm{N}=\mathrm{C}$ & $1.08 \times 10^{4}$ \\
\hline $\operatorname{CCC}(=\mathrm{O}) \mathrm{NC}(=\mathrm{O}) \mathrm{N}=\mathrm{C}$ & $3.05 \times 10^{3}$ \\
\hline CCCN & $5.42 \times 10^{2}$ \\
\hline $\mathrm{CCC}(\mathrm{N}) \mathrm{O}$ & $1.15 \times 10^{4}$ \\
\hline $\operatorname{CCC}(\mathrm{O}) \mathrm{NC}(=\mathrm{O}) \mathrm{N}=\mathrm{C}$ & $2.45 \times 10^{4}$ \\
\hline $\mathrm{Cc} 1 \mathrm{c}(\mathrm{C}) \mathrm{c}(\mathrm{S}) \mathrm{c}(\mathrm{C}([\mathrm{O}-])=\mathrm{O}) \mathrm{c}(\mathrm{O}) \mathrm{c} 1 \mathrm{O}$ & $6.80 \times 10^{1}$ \\
\hline $\mathrm{Cc} 1 \mathrm{c}(\mathrm{C}) \mathrm{c}(\mathrm{C}([\mathrm{O}-])=\mathrm{O}) \mathrm{c}(\mathrm{O}) \mathrm{c}(\mathrm{O}) \mathrm{c} 1 \mathrm{~S}$ & $6.80 \times 10^{1}$ \\
\hline $\mathrm{Cc} 1 \mathrm{c}(\mathrm{CO}) \mathrm{c}(\mathrm{S}) \mathrm{c}(\mathrm{C}([\mathrm{O}-])=\mathrm{O}) \mathrm{c}(\mathrm{N}) \mathrm{c} 1 \mathrm{C}([\mathrm{O}-])=\mathrm{O}$ & $7.53 \times 10^{3}$ \\
\hline $\mathrm{Cc} 1 \mathrm{c}(\mathrm{S}) \mathrm{c}(\mathrm{C}([\mathrm{O}-])=\mathrm{O}) \mathrm{c}(\mathrm{N}) \mathrm{c}(\mathrm{C}([\mathrm{O}-])=\mathrm{O}) \mathrm{c} 1 \mathrm{CO}$ & $7.53 \times 10^{3}$ \\
\hline $\operatorname{Cc} 1 c(C) c(c(C([\mathrm{O}-])=O) c(N) c 1 C([O-])=O) S([O-])=O$ & $6.29 \times 10^{5}$ \\
\hline $\mathrm{Cc} 1 \mathrm{c}(\mathrm{C}) \mathrm{c}(\mathrm{C}([\mathrm{O}-])=\mathrm{O}) \mathrm{c}(\mathrm{N}) \mathrm{c}(\mathrm{C}([\mathrm{O}-])=\mathrm{O}) \mathrm{c} 1 \mathrm{~S}$ & $4.86 \times 10^{2}$ \\
\hline $\mathrm{Cc} 1 \mathrm{c}(\mathrm{C}) \mathrm{c}(\mathrm{S}) \mathrm{c}(\mathrm{C}([\mathrm{O}-])=\mathrm{O}) \mathrm{c}(\mathrm{O}) \mathrm{c} 1 \mathrm{O}$ & $6.80 \times 10^{1}$ \\
\hline $\mathrm{Cc} 1 \mathrm{c}(\mathrm{C}) \mathrm{c}(\mathrm{C}([\mathrm{O}-])=\mathrm{O}) \mathrm{c}(\mathrm{O}) \mathrm{c}(\mathrm{O}) \mathrm{c} 1 \mathrm{~S}$ & $6.80 \times 10^{1}$ \\
\hline $\mathrm{Cc} 1 \mathrm{c}(\mathrm{CO}) \mathrm{c}(\mathrm{S}) \mathrm{c}(\mathrm{C}([\mathrm{O}-])=\mathrm{O}) \mathrm{c}(\mathrm{N}) \mathrm{c} 1 \mathrm{C}([\mathrm{O}-])=\mathrm{O}$ & $7.53 \times 10^{3}$ \\
\hline $\mathrm{Cc} 1 \mathrm{c}(\mathrm{S}) \mathrm{c}(\mathrm{C}([\mathrm{O}-])=\mathrm{O}) \mathrm{c}(\mathrm{N}) \mathrm{c}(\mathrm{C}([\mathrm{O}-])=\mathrm{O}) \mathrm{c} 1 \mathrm{CO}$ & $7.53 \times 10^{3}$ \\
\hline $\mathrm{Cc} 1 \mathrm{c}(\mathrm{C}) \mathrm{c}(\mathrm{c}(\mathrm{C}([\mathrm{O}-])=\mathrm{O}) \mathrm{c}(\mathrm{N}) \mathrm{c} 1 \mathrm{C}([\mathrm{O}-])=\mathrm{O}) \mathrm{S}([\mathrm{O}-])=\mathrm{O}$ & $6.29 \times 10^{5}$ \\
\hline
\end{tabular}


N-(4-Cyanophenyl)N'-cyano-S-

Nc1ccc(O)cc1

$1.20 \times 10^{3}$

methylisothiourea

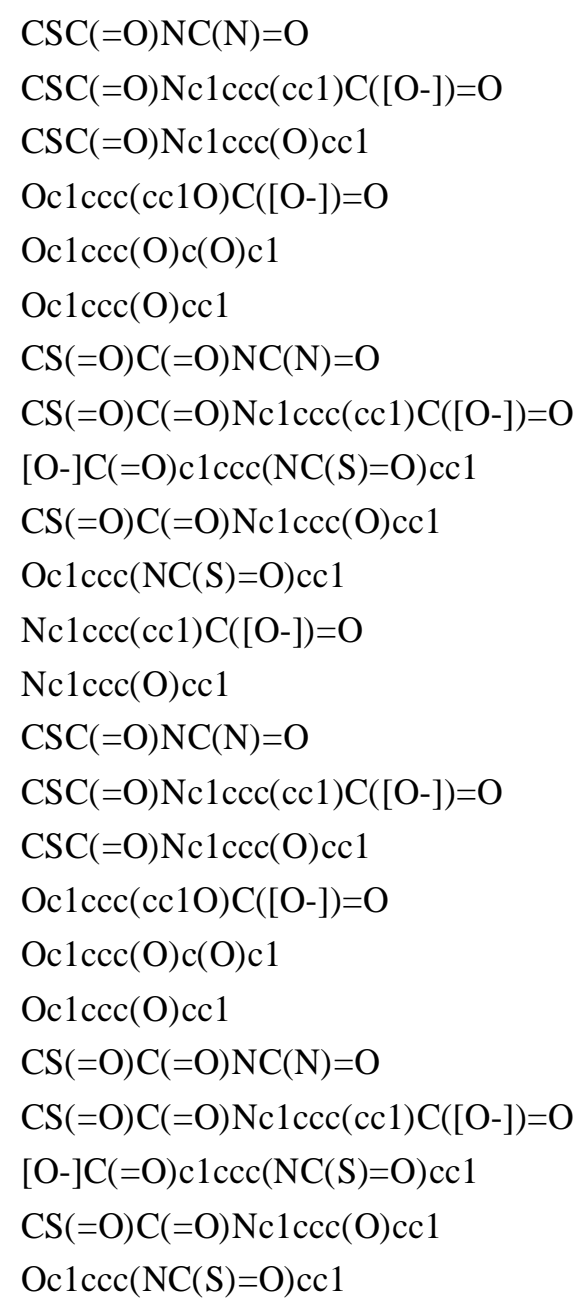

$2.14 \times 10^{3}$

$8.92 \times 10^{1}$

$2.84 \times 10^{2}$

$4.99 \times 10^{2}$

$7.90 \times 10^{2}$

$2.87 \times 10^{2}$

$2.51 \times 10^{5}$

$1.12 \times 10^{5}$

$2.26 \times 10^{2}$

$3.14 \times 10^{4}$

$7.14 \times 10^{2}$

$4.10 \times 10^{2}$

$1.20 \times 10^{3}$

$2.14 \times 10^{3}$

$8.91 \times 10^{1}$

$2.84 \times 10^{2}$

$4.99 \times 10^{2}$

$7.90 \times 10^{2}$

$2.87 \times 10^{2}$

$2.51 \times 10^{5}$

$1.12 \times 10^{5}$

$2.26 \times 10^{2}$

$3.24 \times 10^{4}$

$7.14 \times 10^{2}$

4-Bromo-2-methyl-

Cc1nc2c(Br)c(N)c(O)c(O)c2s1 2.01×10

1,3-benzothiazol-5amine

Nc1ccc2sc(CO)nc2c1Br
Cc1nc2cc(N)ccc2s1
Nc1c(O)c(O)c2sc(CO)nc2c1Br
Cc1nc2cc(N)c(O)c(O)c2s1
Nc1ccc2sc(C=O)nc2c1Br
Nc1ccc2sc(CO)nc2c1
Cc1nc2c(O)c(O)ccc2s1
Nc1c(O)c(O)c2sc(C=O)nc2c1Br
Nc1cc2nc(CO)sc2c(O)c1O
Nc1ccc2sc(nc2c1Br)C([O-])=O
Nc1ccc2sc $(\mathrm{C}=O) n c 2 c 1$
OCc1nc2c(O)c(O)ccc2s1
Cc1nc2c(Br)c(N)ccc2s1
Cc1nc2c(Br $)(N) c(O) c(O) c 2 s 1$

$4.75 \times 10^{2}$

$1.05 \times 10^{2}$

$3.09 \times 10^{3}$

$7.28 \times 10^{2}$

$1.48 \times 10^{2}$

$1.68 \times 10^{3}$

$1.26 \times 10^{2}$

$9.62 \times 10^{2}$

$1.15 \times 10^{4}$

$1.16 \times 10^{2}$

$5.21 \times 10^{2}$

$1.99 \times 10^{3}$

$3.07 \times 10^{1}$

$2.01 \times 10^{2}$ 


\begin{tabular}{|c|c|}
\hline Nc1ccc2sc(CO)nc2c1Br & $4.75 \times 10^{2}$ \\
\hline Cc1nc2cc(N)ccc2s1 & $1.05 \times 10^{2}$ \\
\hline $\mathrm{Nc} 1 \mathrm{c}(\mathrm{O}) \mathrm{c}(\mathrm{O}) \mathrm{c} 2 \mathrm{sc}(\mathrm{CO}) \mathrm{nc} 2 \mathrm{c} 1 \mathrm{Br}$ & $3.09 \times 10^{3}$ \\
\hline Cc1nc2cc $(N) c(O) c(O) c 2 s 1$ & $7.28 \times 10^{2}$ \\
\hline Nc1ccc2sc $(C=O) n c 2 c 1 B r$ & $1.48 \times 10^{2}$ \\
\hline Nc1ccc2sc(CO)nc2c1 & $1.68 \times 10^{3}$ \\
\hline $\mathrm{Cc} 1 \mathrm{nc} 2 \mathrm{c}(\mathrm{O}) \mathrm{c}(\mathrm{O}) \mathrm{ccc} 2 \mathrm{~s} 1$ & $1.26 \times 10^{2}$ \\
\hline $\mathrm{Nc} 1 \mathrm{c}(\mathrm{O}) \mathrm{c}(\mathrm{O}) \mathrm{c} 2 \mathrm{sc}(\mathrm{C}=\mathrm{O}) \mathrm{nc} 2 \mathrm{c} 1 \mathrm{Br}$ & $9.62 \times 10^{2}$ \\
\hline Nc1cc2nc(CO)sc2c(O)c1O & $1.15 \times 10^{4}$ \\
\hline Nc1ccc2sc(nc2c1Br)C([O-])=O & $1.16 \times 10^{2}$ \\
\hline Nc1ccc2sc $(\mathrm{C}=\mathrm{O}) \mathrm{nc} 2 \mathrm{c} 1$ & $5.21 \times 10^{2}$ \\
\hline OCc1nc2c(O)c(O)ccc2s1 & $1.99 \times 10^{3}$ \\
\hline $\mathrm{CC}(\mathrm{C})(\mathrm{O}) \mathrm{CCCCCCCCCCOCCC}[\mathrm{NH} 2+][\mathrm{O}-]$ & $6.02 \times 10^{1}$ \\
\hline CC(C)ССССССССС $(\mathrm{O}) \mathrm{COCCC}[\mathrm{NH} 2+][\mathrm{O}-]$ & $6.42 \times 10^{1}$ \\
\hline CС(C)ССССССССССОСС $(\mathrm{O}) \mathrm{C}[\mathrm{NH} 2+][\mathrm{O}-]$ & $6.42 \times 10^{1}$ \\
\hline $\mathrm{OCCC}[\mathrm{NH} 2+][\mathrm{O}-]$ & $1.83 \times 10^{5}$ \\
\hline$[\mathrm{O}-][\mathrm{NH} 2+] \mathrm{CCC}=\mathrm{O}$ & $1.87 \times 10^{5}$ \\
\hline СС(C)СССССССССС $=\mathrm{O}$ & $2.72 \times 10^{-1}$ \\
\hline $\operatorname{cC}(\mathrm{C}) \operatorname{CCCCCССССС~}([\mathrm{O}-])=\mathrm{O}$ & $1.53 \times 10^{0}$ \\
\hline СС(С)ССССССССССО & $2.19 \times 10^{-1}$ \\
\hline$[\mathrm{O}-][\mathrm{NH} 2+] \mathrm{CCC}([\mathrm{O}-])=\mathrm{O}$ & $1.39 \times 10^{5}$ \\
\hline $\mathrm{CC}(\mathrm{C})(\mathrm{O}) \mathrm{C}(\mathrm{O}) \mathrm{CCCCCCCCCOCCC}[\mathrm{NH} 2+][\mathrm{O}-]$ & $5.03 \times 10^{2}$ \\
\hline $\mathrm{CC}(\mathrm{C})(\mathrm{O}) \mathrm{CCCCССССС(O)COCCC}[\mathrm{NH} 2+][\mathrm{O}-]$ & $5.03 \times 10^{2}$ \\
\hline $\mathrm{CC}(\mathrm{C})(\mathrm{O}) \mathrm{CCCCCCССССОСС(O)C[NH2+][O-]}$ & $5.03 \times 10^{2}$ \\
\hline CC(C)ССССССССC $(=0)$ COCCC $[\mathrm{NH} 2+][\mathrm{O}-]$ & $6.52 \times 10^{1}$ \\
\hline CС(C)ССССССССССОСС $(=0) \mathrm{C}[\mathrm{NH} 2+][\mathrm{O}-]$ & $1.62 \times 10^{1}$ \\
\hline CC(C)ССССССССССОССС $(\mathrm{O})[\mathrm{NH} 2+][\mathrm{O}-]$ & $6.43 \times 10^{1}$ \\
\hline $\operatorname{cC}(\mathrm{C}) \operatorname{CccccccC}([\mathrm{O}-])=\mathrm{O}$ & $8.39 \times 10^{0}$ \\
\hline $\mathrm{OC}(\mathrm{C}[\mathrm{NH} 2+][\mathrm{O}-]) \mathrm{C}([\mathrm{O}-])=\mathrm{O}$ & $1.51 \times 10^{5}$ \\
\hline $\mathrm{CC}(\mathrm{C})(\mathrm{O}) \mathrm{CCCCCССССС}=\mathrm{O}$ & $4.58 \times 10^{0}$ \\
\hline $\operatorname{CC}(\mathrm{C})(\mathrm{O}) \operatorname{CCCCCCCCCC}([\mathrm{O}-])=\mathrm{O}$ & $2.75 \times 10^{1}$ \\
\hline СС(C)(O)ССССССССССО & $1.85 \times 10^{0}$ \\
\hline $\mathrm{CC}(\mathrm{C})(\mathrm{O}) \mathrm{C}(=\mathrm{O}) \mathrm{CCCCCCCCCOCCC}[\mathrm{NH} 2+][\mathrm{O}-]$ & $4.99 \times 10^{2}$ \\
\hline $\mathrm{CC}(\mathrm{C})(\mathrm{O}) \mathrm{CCCCCCCCC}(=\mathrm{O}) \mathrm{COCCC}[\mathrm{NH} 2+][\mathrm{O}-]$ & $1.07 \times 10^{3}$ \\
\hline $\mathrm{CC}(\mathrm{C})(\mathrm{O}) \mathrm{CCCCССССССОСС}(=\mathrm{O}) \mathrm{C}[\mathrm{NH} 2+][\mathrm{O}-]$ & $2.66 \times 10^{2}$ \\
\hline CС $(\mathrm{C})(\mathrm{O}) \mathrm{CСССССССССОССС}(\mathrm{O})[\mathrm{NH} 2+][\mathrm{O}-]$ & $5.03 \times 10^{2}$ \\
\hline $\mathrm{CC}(\mathrm{C}) \mathrm{CCCCCCCC}(\mathrm{O}) \mathrm{C}(=\mathrm{O}) \mathrm{COCCC}[\mathrm{NH} 2+][\mathrm{O}-]$ & $1.10 \times 10^{2}$ \\
\hline CС(C)ССССССССС $(=O) \operatorname{COCC}(O) C[N H 2+][O-]$ & $1.14 \times 10^{3}$ \\
\hline $\mathrm{CC}(\mathrm{C}) \operatorname{CCCCCCCСС~}(\mathrm{O}) \mathrm{C}=\mathrm{O}$ & $4.90 \times 10^{0}$ \\
\hline $\operatorname{CC}(\mathrm{C}) \operatorname{CCCCCCCCC~}(\mathrm{O}) \mathrm{C}([\mathrm{O}-])=\mathrm{O}$ & $1.64 \times 10^{1}$ \\
\hline СС(С)ССССССССС $(\mathrm{O}) \mathrm{CO}$ & $1.98 \times 10^{0}$ \\
\hline $\operatorname{CC}(\mathrm{C}) \operatorname{CCCCCСССС~}(=\mathrm{O}) \mathrm{CO}$ & $2.23 \times 10^{0}$ \\
\hline
\end{tabular}




\begin{tabular}{|c|c|}
\hline CС $(\mathrm{C}) \operatorname{CСССССССС~}(\mathrm{O}) \operatorname{COCC}(=\mathrm{O}) \mathrm{C}[\mathrm{NH} 2+][\mathrm{O}-]$ & $2.84 \times 10^{2}$ \\
\hline $\mathrm{OCC}(\mathrm{O}) \mathrm{C}[\mathrm{NH} 2+][\mathrm{O}-]$ & $1.70 \times 10^{6}$ \\
\hline $\mathrm{OC}(\mathrm{C}[\mathrm{NH} 2+][\mathrm{O}-]) \mathrm{C}=\mathrm{O}$ & $3.67 \times 10^{6}$ \\
\hline $\mathrm{OCC}(=\mathrm{O}) \mathrm{C}[\mathrm{NH} 2+][\mathrm{O}-]$ & $4.15 \times 10^{6}$ \\
\hline $\mathrm{OCCC}(\mathrm{O})[\mathrm{NH} 2+][\mathrm{O}-]$ & $1.70 \times 10^{6}$ \\
\hline СС(С)ССССССССССОССС $=\mathrm{O}$ & $2.37 \times 10^{-1}$ \\
\hline $\mathrm{CC}(\mathrm{C})(\mathrm{O}) \mathrm{C}(\mathrm{O}) \mathrm{CCССССССС}=\mathrm{O}$ & $3.90 \times 10^{1}$ \\
\hline $\mathrm{CC}(\mathrm{C})(\mathrm{O}) \mathrm{C}(\mathrm{O}) \mathrm{CCСССССССО}$ & $3.57 \times 10^{1}$ \\
\hline $\mathrm{CC}(\mathrm{C})(\mathrm{O}) \operatorname{CCCCCСССС~}(\mathrm{O}) \mathrm{C}=\mathrm{O}$ & $3.90 \times 10^{1}$ \\
\hline $\mathrm{CC}(\mathrm{C})(\mathrm{O}) \mathrm{CCCCССССС}(\mathrm{O}) \mathrm{CO}$ & $3.57 \times 10^{1}$ \\
\hline $\operatorname{cC}(\mathrm{C}) \operatorname{CcccccсCC}(=\mathrm{O}) \mathrm{C}=\mathrm{O}$ & $8.13 \times 10^{0}$ \\
\hline$[\mathrm{O}-][\mathrm{NH} 2+] \mathrm{CC}(=\mathrm{O}) \mathrm{C}=\mathrm{O}$ & $6.03 \times 10^{6}$ \\
\hline CС(C)ССССССССССОССС[NH2+][O-] & $3.65 \times 10^{0}$ \\
\hline $\mathrm{CC}(\mathrm{C})(\mathrm{O}) \mathrm{CCCCCCСCCCOCCC}[\mathrm{NH} 2+][\mathrm{O}-]$ & $6.02 \times 10^{1}$ \\
\hline CC(C)ССССССССС $(\mathrm{O}) \mathrm{COCCC}[\mathrm{NH} 2+][\mathrm{O}-]$ & $6.43 \times 10^{1}$ \\
\hline СС(C)ССССССССССОСС $(\mathrm{O}) \mathrm{C}[\mathrm{NH} 2+][\mathrm{O}-]$ & $6.43 \times 10^{1}$ \\
\hline OCCC $[\mathrm{NH} 2+][\mathrm{O}-]$ & $1.83 \times 10^{5}$ \\
\hline$[\mathrm{O}-][\mathrm{NH} 2+] \mathrm{CCC}=\mathrm{O}$ & $1.87 \times 10^{5}$ \\
\hline $\mathrm{CC}(\mathrm{C}) \mathrm{CССССССССС}=\mathrm{O}$ & $2.72 \times 10^{-1}$ \\
\hline $\operatorname{cC}(\mathrm{C}) \operatorname{CCCCCCCСCC~}([\mathrm{O}-])=\mathrm{O}$ & $1.53 \times 10^{0}$ \\
\hline СС(С)ССССССССССО & $2.19 \times 10^{-1}$ \\
\hline$[\mathrm{O}-][\mathrm{NH} 2+] \mathrm{CCC}([\mathrm{O}-])=\mathrm{O}$ & $1.39 \times 10^{5}$ \\
\hline $\mathrm{CC}(\mathrm{C})(\mathrm{O}) \mathrm{C}(\mathrm{O}) \mathrm{CCCCCCCCCOCCC}[\mathrm{NH} 2+][\mathrm{O}-]$ & $5.03 \times 10^{2}$ \\
\hline CC(C)(O)CСССССССС $(\mathrm{O}) \mathrm{COCCC}[\mathrm{NH} 2+][\mathrm{O}-]$ & $5.03 \times 10^{2}$ \\
\hline $\mathrm{CC}(\mathrm{C})(\mathrm{O}) \mathrm{CCCСССССССОСС(O)C[NH2+][O-]}$ & $5.03 \times 10^{2}$ \\
\hline CC(C)ССССССССС $(=O)$ COCСC[NH2+][O-] & $6.52 \times 10^{1}$ \\
\hline CС(C)ССССССССССОСС $(=0) C[\mathrm{NH} 2+][\mathrm{O}-]$ & $1.62 \times 10^{1}$ \\
\hline CС(C)ССССССССССОССС(O)[NH2+][O-] & $6.43 \times 10^{1}$ \\
\hline $\operatorname{CC}(\mathrm{C}) \operatorname{CCCCCCCC~}([\mathrm{O}-])=\mathrm{O}$ & $8.39 \times 10^{0}$ \\
\hline $\mathrm{OC}(\mathrm{C}[\mathrm{NH} 2+][\mathrm{O}-]) \mathrm{C}([\mathrm{O}-])=\mathrm{O}$ & $1.51 \times 10^{6}$ \\
\hline $\mathrm{CC}(\mathrm{C})(\mathrm{O}) \mathrm{CCСССССССС}=\mathrm{O}$ & $4.58 \times 10^{0}$ \\
\hline $\operatorname{CC}(\mathrm{C})(\mathrm{O}) \operatorname{CCCCCCCCCC~}([\mathrm{O}-])=\mathrm{O}$ & $2.75 \times 10^{1}$ \\
\hline CС $(\mathrm{C})(\mathrm{O}) \mathrm{CCCСССССССО}$ & $1.85 \times 10^{0}$ \\
\hline $\mathrm{CC}(\mathrm{C})(\mathrm{O}) \mathrm{C}(=\mathrm{O}) \mathrm{CCCCCCCCCOCCC}[\mathrm{NH} 2+][\mathrm{O}-]$ & $4.99 \times 10^{2}$ \\
\hline $\mathrm{CC}(\mathrm{C})(\mathrm{O}) \operatorname{CCCCCСССС~}(=\mathrm{O}) \mathrm{COCCC}[\mathrm{NH} 2+][\mathrm{O}-]$ & $1.07 \times 10^{3}$ \\
\hline $\mathrm{CC}(\mathrm{C})(\mathrm{O}) \mathrm{CCCCCCCCCCOCC}(=\mathrm{O}) \mathrm{C}[\mathrm{NH} 2+][\mathrm{O}-]$ & $2.66 \times 10^{2}$ \\
\hline CС(C)(O)ССССССССССОССС $(\mathrm{O})[\mathrm{NH} 2+][\mathrm{O}-]$ & $5.03 \times 10^{2}$ \\
\hline CC(C)CССССССС $(\mathrm{O}) \mathrm{C}(=\mathrm{O}) \mathrm{COCCC}[\mathrm{NH} 2+][\mathrm{O}-]$ & $1.10 \times 10^{2}$ \\
\hline СС(C)ССССССССС $(=0) \operatorname{COCC}(\mathrm{O}) \mathrm{C}[\mathrm{NH} 2+][\mathrm{O}-]$ & $1.14 \times 10^{3}$ \\
\hline $\mathrm{CC}(\mathrm{C}) \operatorname{CСССССССС~}(\mathrm{O}) \mathrm{C}=\mathrm{O}$ & $4.90 \times 10^{0}$ \\
\hline $\operatorname{cC}(\mathrm{C}) \operatorname{CCCCCCCCC~}(\mathrm{O}) \mathrm{C}([\mathrm{O}-])=\mathrm{O}$ & $1.64 \times 10^{1}$ \\
\hline СС (C)ССССССССС $(\mathrm{O}) \mathrm{CO}$ & $1.98 \times 10^{0}$ \\
\hline $\mathrm{CC}(\mathrm{C}) \operatorname{CCCСССССС~}(=\mathrm{O}) \mathrm{CO}$ & $2.23 \times 10^{0}$ \\
\hline 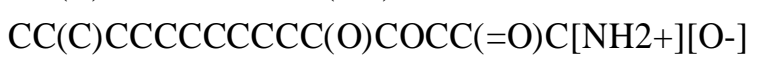 & $2.84 \times 10^{2}$ \\
\hline
\end{tabular}




\begin{tabular}{|c|c|c|}
\hline & $\mathrm{OCC}(\mathrm{O}) \mathrm{C}[\mathrm{NH} 2+][\mathrm{O}-]$ & $1.70 \times 10^{6}$ \\
\hline & $\mathrm{OC}(\mathrm{C}[\mathrm{NH} 2+][\mathrm{O}-]) \mathrm{C}=\mathrm{O}$ & $3.67 \times 10^{6}$ \\
\hline & $\mathrm{OCC}(=\mathrm{O}) \mathrm{C}[\mathrm{NH} 2+][\mathrm{O}-]$ & $4.15 \times 10^{5}$ \\
\hline & $\mathrm{OCCC}(\mathrm{O})[\mathrm{NH} 2+][\mathrm{O}-]$ & $1.70 \times 10^{6}$ \\
\hline & СС $($ С) ССССССССССОССС $=\mathrm{O}$ & $2.37 \times 10^{-1}$ \\
\hline & $\mathrm{CC}(\mathrm{C})(\mathrm{O}) \mathrm{C}(\mathrm{O}) \mathrm{CСССССССС}=\mathrm{O}$ & $3.90 \times 10^{1}$ \\
\hline & $\mathrm{CC}(\mathrm{C})(\mathrm{O}) \mathrm{C}(\mathrm{O}) \mathrm{CССССССССО}$ & $3.57 \times 10^{1}$ \\
\hline & $\operatorname{cC}(\mathrm{C})(\mathrm{O}) \operatorname{CCCСССССС~}(\mathrm{O}) \mathrm{C}=\mathrm{O}$ & $3.90 \times 10^{1}$ \\
\hline & $\mathrm{CC}(\mathrm{C})(\mathrm{O}) \mathrm{CCССССССС}(\mathrm{O}) \mathrm{CO}$ & $3.57 \times 10^{1}$ \\
\hline & $\operatorname{CC}(\mathrm{C}) \operatorname{CCCCCCCCC~}(=\mathrm{O}) \mathrm{C}=\mathrm{O}$ & $8.13 \times 10^{0}$ \\
\hline & {$[\mathrm{O}-][\mathrm{NH} 2+] \mathrm{CC}(=\mathrm{O}) \mathrm{C}=\mathrm{O}$} & $6.03 \times 10^{6}$ \\
\hline 2-Amino-4- & $\mathrm{Cc} 1 \mathrm{cc}(\mathrm{O}) \mathrm{c}(\mathrm{O}) \mathrm{c} 2 \mathrm{sc}(\mathrm{N}) \mathrm{nc} 12$ & $1.85 \times 10^{2}$ \\
\hline & Nc1nc2c(CO)cccc2s1 & $4.27 \times 10^{2}$ \\
\hline & $\mathrm{C} \| \mathrm{C}(=\mathrm{C} \| \mathrm{C}([\mathrm{O}-])=\mathrm{O}) \mathrm{c} 1 \mathrm{nc}(\mathrm{N}) \operatorname{sc} 1 \mathrm{C}([\mathrm{O}-])=\mathrm{O}$ & $3.44 \times 10^{2}$ \\
\hline & Nc1nc2c $(C=0) \operatorname{cccc} 2 s 1$ & $1.32 \times 10^{2}$ \\
\hline & Nc1nc2c(CO)cc(O)c(O)c2s1 & $2.91 \times 10^{3}$ \\
\hline & $\mathrm{CC}(\mathrm{O})(\mathrm{CC}([\mathrm{O}-])=\mathrm{O}) \operatorname{c} 1 \mathrm{nc}(\mathrm{N}) \operatorname{sc} 1 \mathrm{C}([\mathrm{O}-])=\mathrm{O}$ & $4.94 \times 10^{3}$ \\
\hline & $\mathrm{C} \| \mathrm{C}(=\mathrm{C} \| \mathrm{C}([\mathrm{O}-])=\mathrm{O}) \mathrm{c} 1 \csc (\mathrm{N}) \mathrm{n} 1$ & $2.23 \times 10^{2}$ \\
\hline & $\mathrm{C} \| \mathrm{C}(=\mathrm{C} \| \mathrm{C}([\mathrm{O}-])=\mathrm{O}) \mathrm{c} 1 \mathrm{nc}(\mathrm{N}) \mathrm{s}(=\mathrm{O}) \mathrm{c} 1 \mathrm{C}([\mathrm{O}-])=\mathrm{O}$ & $7.75 \times 10^{2}$ \\
\hline & $\mathrm{CC}(\mathrm{CC}([\mathrm{O}-])=\mathrm{O}) \mathrm{c} 1 \mathrm{nc}(\mathrm{N}) \operatorname{sc} 1 \mathrm{C}([\mathrm{O}-])=\mathrm{O}$ & $2.97 \times 10^{2}$ \\
\hline & Nc1nc2c $(\operatorname{cccc} 2 s 1) \mathrm{C}([\mathrm{O}-])=\mathrm{O}$ & $1.07 \times 10^{2}$ \\
\hline & $\mathrm{Nc} 1 \mathrm{nc} 2 \mathrm{c}(\mathrm{C}=\mathrm{O}) \mathrm{cc}(\mathrm{O}) \mathrm{c}(\mathrm{O}) \mathrm{c} 2 \mathrm{~s} 1$ & $9.03 \times 10^{2}$ \\
\hline & Cc1cccc2sc(N)nc12 & $2.68 \times 10^{1}$ \\
\hline & $\mathrm{Cc} 1 \mathrm{cc}(\mathrm{O}) \mathrm{c}(\mathrm{O}) \mathrm{c} 2 \mathrm{sc}(\mathrm{N}) \mathrm{nc} 12$ & $1.85 \times 10^{2}$ \\
\hline & Nc1nc2c(CO)cccc2s1 & $4.27 \times 10^{2}$ \\
\hline & $\mathrm{C} \| \mathrm{C}(=\mathrm{C} \| \mathrm{C}([\mathrm{O}-])=\mathrm{O}) \mathrm{c} 1 \mathrm{nc}(\mathrm{N}) \operatorname{sc} 1 \mathrm{C}([\mathrm{O}-])=\mathrm{O}$ & $3.44 \times 10^{2}$ \\
\hline & Nc1nc2c $(C=0) \operatorname{cccc} 2 s 1$ & $1.32 \times 10^{2}$ \\
\hline & Nc1nc2c(CO)cc(O)c(O)c2s1 & $2.91 \times 10^{3}$ \\
\hline & $\mathrm{CC}(\mathrm{O})(\mathrm{CC}([\mathrm{O}-])=\mathrm{O}) \mathrm{c} 1 \mathrm{nc}(\mathrm{N}) \operatorname{sc} 1 \mathrm{C}([\mathrm{O}-])=\mathrm{O}$ & $4.94 \times 10^{3}$ \\
\hline & $\mathrm{C} \| \mathrm{C}(=\mathrm{C} \| \mathrm{C}([\mathrm{O}-])=\mathrm{O}) \mathrm{c} 1 \csc (\mathrm{N}) \mathrm{n} 1$ & $2.23 \times 10^{2}$ \\
\hline & $\mathrm{C} \| \mathrm{C}(=\mathrm{C} \| \mathrm{C}([\mathrm{O}-])=\mathrm{O}) \mathrm{c} 1 \mathrm{nc}(\mathrm{N}) \mathrm{s}(=\mathrm{O}) \mathrm{c} 1 \mathrm{C}([\mathrm{O}-])=\mathrm{O}$ & $7.75 \times 10^{2}$ \\
\hline & $\mathrm{CC}(\mathrm{CC}([\mathrm{O}-])=\mathrm{O}) \mathrm{c} 1 \mathrm{nc}(\mathrm{N}) \operatorname{sc} 1 \mathrm{C}([\mathrm{O}-])=\mathrm{O}$ & $2.97 \times 10^{2}$ \\
\hline & Nc1nc2c(cccc2s1)C([O-])=O & $1.07 \times 10^{2}$ \\
\hline & Nc1nc2c $(\mathrm{C}=\mathrm{O}) \mathrm{cc}(\mathrm{O}) \mathrm{c}(\mathrm{O}) \mathrm{c} 2 \mathrm{~s} 1$ & $9.03 \times 10^{2}$ \\
\hline
\end{tabular}


Table S7 Comparison of experimental acute toxicity of key toxicants to previous studies.

\begin{tabular}{cccc}
\hline Chemical Name & $\begin{array}{c}\text { 24h-EC50 }(\mathrm{mg} / \mathrm{L}) \\
\text { in Present Study }\end{array}$ & $\begin{array}{c}\text { 24h-EC50 }(\mathrm{mg} / \mathrm{L}) \text { in } \\
\text { Previous Study }\end{array}$ & References \\
\hline Propiconazole & 6.32 & 9.50 & 4 \\
Tebuconazole & 8.47 & 11.59 & 5 \\
Hexaconazole & 6.87 & $6.38 \pm 0.58$ & 6 \\
Flutriafol & 85.70 & $61-78$ & 7 \\
\hline
\end{tabular}




\section{References}

1. EPA, Algal Assay Procedures Bottle Test. National Eutrophication Research Program, Pacific Northwest Environmental; Research Lab, Corvallis, OR (1971). 1971.

2. OECD, Fish embryo toxicity (FET) test. Draft OECD guideline for the testing of chemicals. 2006.

3. ISO, E., Water quality—Determination of the inhibition of the mobility of Daphnia magna Straus (Cladocera, Crustacea)—Acute toxicity test. EN ISO 1998, 6341, 1996.

4. Ochoa-Acuña, H. G.; Bialkowski, W.; Yale, G.; Hahn, L., Toxicity of soybean rust fungicides to freshwater algae and Daphnia magna. Ecotoxicology 2009, 18, (4), 440-446.

5. Sun, M., Study on Environmental Behavior of Four Triazole Chiral Pesticide. 2014.

6. Liang, H., Study on Environmental Behavior and Aquatic Biological Toxicity of Enantiomers of Several Typical Chiral Pesticides. 2014.

7. Ríhová Ambrožová, J., The pesticides in waterbodies and the need of toxicity testing. Journal of Faculty of Ecology and Environmental Sciences Technical University in Zvolen 2008. 